Research Article

\title{
Evolution of Periodic Orbits within the Frame of Formation Satellites
}

\author{
Elbaz I. Abouelmagd (D), ${ }^{1}$ Mitali J. Doshi $\mathbb{D}^{2}{ }^{2}$ and Niraj M. Pathak ${ }^{2}{ }^{2}$ \\ ${ }^{1}$ Celestial Mechanics and Space Dynamics Research Group (CMSDRG), Astronomy Department, \\ National Research Institute of Astronomy and Geophysics (NRIAG), Helwan 11421, Cairo, Egypt \\ ${ }^{2}$ Department of Mathematics, Faculty of Technology, Dharmsinh Desai University, Nadiad, Gujarat 3870001, India
}

Correspondence should be addressed to Elbaz I. Abouelmagd; elbaz.abouelmagd@nriag.sci.eg

Received 20 July 2020; Revised 20 November 2020; Accepted 30 November 2020; Published 21 December 2020

Academic Editor: Juan L. G. Guirao

Copyright (c) 2020 Elbaz I. Abouelmagd et al. This is an open access article distributed under the Creative Commons Attribution License, which permits unrestricted use, distribution, and reproduction in any medium, provided the original work is properly cited.

\begin{abstract}
In the framework of formation satellites, the periodic orbits of deputy satellite are analyzed when the chief satellite is moving in an elliptical orbit. This analysis is developed on 1- to 10-loop periodic orbits of the deputy satellite. These orbits along with their associated loops are discussed under some specific initial position sets. The effects of different initial velocities, initial true anomalies, and eccentricities on the initial position and orbital period of periodic orbits of deputy satellite are investigated.
\end{abstract}

\section{Introduction}

The periodic orbits have substantial and leading role in exploring and understanding the behavior of dynamical systems. At most, they define strange attractors, which lead to chaotic dynamical systems. The special solution of a dynamical system, which repeats and generates itself in time, is called periodic orbit. From the mathematical point of view, the orbit is a set of points associated by the evolution function of the proposed dynamical system. These points are considered as a subset from the phase space, which are covered by the dynamical system trajectory within frame of a particular set from the initial conditions. Some recent works, analyzing the periodic orbits, are addressed in [1-4].

The sufficient condition for the existence of periodic orbits is given when the Hamilton system is a function in the action-angle variables; further, these obtained results are applied to Hamiltonian of the perturbed Kepler problem in [5]. Also, a geometric approach to asymptotically stabilize with a phase of fixed periodic orbits for global Hamiltonian dynamical system is established in [6]. While in [7], the new families of periodic orbits analytically for the Hamilton system are found, which characterize the local motion in the region around the galaxy center. Furthermore, in [8], the theory of averaging is applied to prove the existence of twelve families of periodic orbits in a 3-dimension for a galactic Hamiltonian dynamical system. Since we are interested to evaluate the periodic orbits within frame of formation satellites, we will give also an overview about the literatures and importance of formation satellites in the following paragraphs.

The formation flying of small multiple satellites as a replacement of using single large satellite has shown great interest for different defense- and science-based space missions. Formation flying consists of a set of satellites, which have the same dynamic state and governed by one control law. Abundance and precision of the proposed system in terms of formation satellites are more effective tools, which give a job more accuracy than using a conventional large single satellite. It also reduces the maintenance and launching costs, extremely expands the surveillance area, and gives more resilience into the design of space mission. For example, a sensor of ground observation can be loaded on bunch of satellites flying in a specified formation for increasing aperture size instead of constructing a large single satellite with more expense. There are chances of aborting the whole mission in the event of satellite failure. Proper management of satellites cluster with special planning and scheduling reduces the chances of failure. 
Using formation satellite in space-based missions has many advantages, but at the expense of increased complexity and different challenges like high-precision relative navigation [9], distributed communication [10], stable formation design [11], trajectory optimization and control [12], and attitude synchronization [13]. In formation satellite, trajectory optimization and control problem are two important tasks to achieve a successful launching of satellites set in the space. These tasks comprise maintaining the small satellites in a stable formation within frame of enough accuracy against different perturbations of orbit and maneuvering of formation for guiding and performing control command for reconfiguring from perturbed satellite formation to one stable formation.

The precise model of relative motion in order to analyze satellites formation flying is a basic need which covers accurate linear and nonlinear satellites models of relative motion taking into account $J_{2}$ perturbations. Different relative dynamic models are proposed in the literature using different assumptions with many methodologies. It is necessary to make a comparative study to choose appropriate models for specified missions with perturbation that should be considered for definite applications.

A considerable work is accomplished into satellite formation flying for libration point mission with different models that characterize the relative motion satellites between two or more in low Earth orbit (LEO). The major fundamental of this work is carried out by Hill in [14]. While the relative motion within frame of Clohessy-Wiltshire equations is written in terms of a Cartesian or curvilinear coordinates tracing a circular reference orbit around the Earth and models by using orbit elements differences to characterize relative orbits [15]. The extended version of the Hill equations was given in [16] that involves the influent of the zonal harmonic parameter $J_{2}$ using a force gradient method to time-varying form. It was verified and applied to linear quadratic regulator design and evaluated for the station-keeping task in [17].

In [18], the force gradient modelling approach for satellite formation flying around the libration point $L_{2}$ using periodic halo motion as a reference is investigated. The optimal maneuver problem can be characterized as a state transition problem based on Hill's system and maximum principle of Pontryagin. The optimal solution can be obtained by solving the state transition equations and performing the simulation study [19]. In a formation satellite, a magnetic field approach helps a large number of closely located satellites in tracking each other in six degrees of freedom without disturbing their positions and orientation relative to each other (see [20, 21] for details).

The relative motion control is an important task required in the formation of the flying missions. Different control methods without fuel consumption are of a specific interest. A number of these methods based on atmosphere drag effects, electrostatic magnetic field, and the Lorentz force have been proposed, but exchanging mass between satellites is a novel technique for formation flying relative motion control [22].

This paper is organized into four sections. The importance and applications of formation satellites are discussed in Section 1 as a part of literature review. Model description and derived governing equations of motion are covered in Section 2. Analysis of the given sets of initial positions for deputy satellite, which generate periodic orbits, is investigated in Section 3. While in Section 4, we compare the effect of variation in eccentricity of chief satellite's orbit on periodic orbits of deputy satellite with number of loops. Finally, conclusion is drawn from the analysis becomes the part of Section 5 .

\section{Model Description}

Consider two spacecrafts orbiting a common primary and its mass is $m$. Mainly, the motion of these two spacecrafts is governed by the Kepler model or the dynamical system of two bodies [23-26]. One of the spacecraft is termed as a chief satellite, and the second is referred as a deputy satellite, where their masses are $m_{0}$ and $m_{1}$, respectively. Then, the equations of relative motion of deputy satellite with respect to chief satellite under the setup of the Keplerian two-body problem are obtained as follows: we consider a chief-fixed, local vertical local horizontal (LVLH) rotating frame, also known as EulerHill frame. Here, the origin is located at the position of chief satellite, as shown in Figure 1.

From two-body problem, the motion of the chief and deputy satellites around the primary (Earth or any planet) in inertial frame of reference are given by

$$
\begin{aligned}
& \ddot{\mathbf{r}}_{0}=-\mu_{0} \frac{\mathbf{r}_{0}}{r_{0}^{3}}, \\
& \ddot{\mathbf{r}}_{1}=-\mu_{1} \frac{\mathbf{r}_{1}}{r_{1}^{3}},
\end{aligned}
$$

where $\mu_{0}=m+m_{0}$ and $\mu_{1}=m+m_{1}$, but $m_{0}, m_{1} \ll m$; then, we can approximate $\mu_{0} \approx \mu_{1}=\mu$. Thereby, the solutions of equation (1) are controlled by

$$
\begin{aligned}
& r_{0}=\frac{a_{0}\left(1-e_{0}^{2}\right)}{\left(1+e_{0} \cos f_{0}\right)}, \\
& r_{1}=\frac{a_{1}\left(1-e_{1}^{2}\right)}{\left(1+e_{1} \cos f_{1}\right)},
\end{aligned}
$$

where $a_{0}\left(a_{1}\right), e_{0}\left(e_{1}\right)$, and $f_{0}\left(f_{1}\right)$ are the semimajor axis, eccentricity, and true anomaly of chief (deputy) satellite's orbit, respectively.

Now, we assume that $\rho$ is the position vector of deputy satellite relative to chief satellite; hence, $\boldsymbol{\rho}=\mathbf{r}_{1}-\mathbf{r}_{0}$, and the relative motion of deputy satellite is

$$
\ddot{\boldsymbol{\rho}}=-\mu \frac{\mathbf{r}_{0}+\boldsymbol{\rho}}{\left|\mathbf{r}_{0}+\boldsymbol{\rho}\right|^{3}}+\mu \frac{\mathbf{r}_{0}}{r_{0}^{3}},
$$

where $\mathbf{r}_{0}=\left[r_{0}, 0,0\right]^{T}, \boldsymbol{\rho}=[x, y, z]^{T}, f_{0}=\theta_{0}-\omega$, and $\omega$ is the argument of periapsis. But $\omega$ is a constant. Thereby, we can define the angular velocity vector $\boldsymbol{\Omega}=\left[0,0, \dot{\theta}_{0}\right]^{T}$.

The general relation between the velocity and acceleration in the inertial frame and the rotating by the angular velocity $\Omega$ is controlled by 


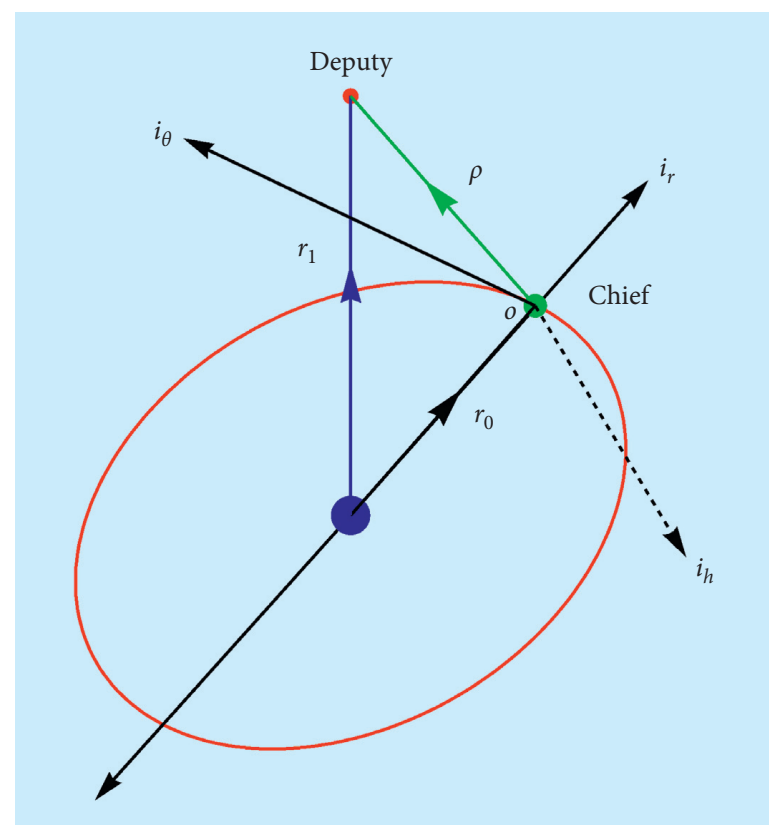

Figure 1: Configuration of the LVLH frame.

$$
\begin{aligned}
& \dot{\boldsymbol{\rho}}^{\mathscr{I}}=\dot{\boldsymbol{\rho}}^{\mathscr{R}}+\boldsymbol{\Omega} \wedge \boldsymbol{\rho}, \\
& \ddot{\boldsymbol{\rho}}^{\mathscr{I}}=\ddot{\boldsymbol{\rho}}^{\mathscr{R}}+2 \boldsymbol{\Omega} \wedge \dot{\boldsymbol{\rho}}^{\mathscr{R}}+\boldsymbol{\Omega} \wedge(\boldsymbol{\Omega} \wedge \boldsymbol{\rho})+\dot{\boldsymbol{\Omega}} \wedge \boldsymbol{\rho},
\end{aligned}
$$

where

$$
\begin{aligned}
& \dot{\boldsymbol{\rho}}^{\mathscr{R}}=\dot{x} i_{r}+\dot{y} i_{\theta}+\ddot{z} i_{h}, \\
& \ddot{\boldsymbol{\rho}}^{\mathscr{R}}=\ddot{x} i_{r}+\ddot{y} i_{\theta}+\ddot{z} i_{h} .
\end{aligned}
$$

Utilizing equations (3)-(5) componentwise equations of relative motion are given by

$$
\begin{aligned}
& \ddot{x}-2 \dot{\theta}_{0} \dot{y}-\ddot{\theta}_{0} y-\dot{\theta}_{0}^{2} x=-\left(r_{0}+x\right) F\left(x, y, z, r_{0}\right)+G\left(r_{0}\right), \\
& \ddot{y}+2 \dot{\theta}_{0} \dot{x}+\ddot{\theta}_{0} x-\dot{\theta}_{0}^{2} y=-y F\left(x, y, z, r_{0}\right), \ddot{z},=-z F\left(x, y, z, r_{0}\right),
\end{aligned}
$$

where

$$
\begin{gathered}
F\left(x, y, z, r_{0}\right)=\frac{\mu}{\left[\left(r_{0}+x\right)^{2}+y^{2}+z^{2}\right]^{3 / 2}}, \\
G\left(r_{0}\right)=\frac{\mu}{r_{0}^{2}} .
\end{gathered}
$$

The system of equation (6) represents the general relative motion with respect to independent time variable. In order to have docile analysis for these equations, we will change the independent time variable by the true anomaly and scale the relative positions by the chief satellite radius. Thereby, we take $\bar{x}=x / r_{0}, \bar{y}=y / r_{0}, \bar{z}=z / r_{0}$; since $v=\sqrt{\dot{\mathbf{r}} . \dot{\mathbf{r}}}$ is the velocity, $h=r_{0}^{2} \dot{\theta}_{0}=r_{0}^{2} \dot{f}_{0}$ and $P=h^{2} / \mu$ is the semilatus rectum; then with help of equation (2), the velocity and accelerations components are controlled by

$$
\begin{aligned}
\dot{x} & =\frac{h_{0}}{P_{0}}\left[\left(1+e_{0} \cos f_{0}\right) \frac{\mathrm{d} \bar{x}}{\mathrm{~d} f_{0}}+\bar{x}\left(e_{0} \sin f_{0}\right)\right], \\
\dot{y} & =\frac{h_{0}}{P_{0}}\left[\left(1+e_{0} \cos f_{0}\right) \frac{\mathrm{d} \bar{y}}{\mathrm{~d} f_{0}}+\bar{y}\left(e_{0} \sin f_{0}\right)\right], \\
\dot{z} & =\frac{h_{0}}{P_{0}}\left[\left(1+e_{0} \cos f_{0}\right) \frac{\mathrm{d} \bar{z}}{\mathrm{~d} f_{0}}+\bar{z}\left(e_{0} \sin f_{0}\right)\right], \\
\ddot{x} & =\frac{h_{0}^{2}}{P_{0}^{3}} \Upsilon^{2}\left(f_{0}\right)\left[\Upsilon\left(f_{0}\right) \frac{\mathrm{d}^{2} \bar{x}}{\mathrm{~d} f_{0}^{2}}+\bar{x}\left(e_{0} \cos f_{0}\right)\right], \ddot{y} \\
& =\frac{h_{0}^{2}}{P_{0}^{3}} \Upsilon^{2}\left(f_{0}\right)\left[\Upsilon\left(f_{0}\right) \frac{\mathrm{d}^{2} \bar{y}}{\mathrm{~d} f_{0}^{2}}+\bar{y}\left(e_{0} \cos f_{0}\right)\right], \ddot{z} \\
& =\frac{h_{0}^{2}}{P_{0}^{3}} \Upsilon^{2}\left(f_{0}\right)\left[\Upsilon\left(f_{0}\right) \frac{\mathrm{d}^{2} \bar{z}}{\mathrm{~d} f_{0}^{2}}+\bar{z}\left(e_{0} \cos f_{0}\right)\right],
\end{aligned}
$$

where

$$
\Upsilon\left(f_{0}\right)=\left(1+e_{0} \cos f_{0}\right) .
$$

Substituting equations (8) and (9) into equation (6), we get

$$
\begin{aligned}
\frac{\mathrm{d}^{2} \bar{x}}{\mathrm{~d} f_{0}^{2}}-2 \frac{\mathrm{d} \bar{y}}{\mathrm{~d} f_{0}} & =\frac{1+\bar{x}}{\left(1+e_{0} \cos f_{0}\right)}[1-\Gamma(\bar{x} \\
\frac{\mathrm{d}^{2} \bar{y}}{\mathrm{~d} f_{0}^{2}}+2 \frac{\mathrm{d} \bar{x}}{\mathrm{~d} f_{0}} & =\frac{\bar{y}}{\left(1+e_{0} \cos f_{0}\right)}[1-\Gamma(\bar{x} \\
\frac{\mathrm{d}^{2} \bar{z}}{\mathrm{~d} f_{0}^{2}} & =\frac{-\bar{z}}{\left(1+e_{0} \cos f_{0}\right)}\left[e_{0} \cos f_{0}+\Gamma(\bar{x}, t \bar{y} n, q \bar{z})\right],
\end{aligned}
$$


where

$$
\Gamma(\bar{x}, t \bar{y} n, q \bar{z})=\frac{1}{\left[(1+\bar{x})^{2}+\bar{y}^{2}+\bar{z}^{2}\right]^{3 / 2}} .
$$

We will drop the subscript zero and bar for simplicity, thereby equation (11) can be rewritten with a simple form as

$$
\begin{array}{r}
\frac{\mathrm{d}^{2} x}{\mathrm{~d} f^{2}}-2 \frac{\mathrm{d} y}{\mathrm{~d} f}=\frac{\partial W}{\partial x}, \\
\frac{\mathrm{d}^{2} y}{\mathrm{~d} f^{2}}+2 \frac{\mathrm{d} x}{\mathrm{~d} f}=\frac{\partial W}{\partial y}, \\
\frac{\mathrm{d}^{2} z}{\mathrm{~d} f^{2}}=\frac{\partial W}{\partial z},
\end{array}
$$

where

$$
\begin{aligned}
& W=\frac{1}{1+e \cos f}\left[\frac{1}{2}\left(x^{2}+y^{2}-e z^{2} \cos f\right)-U\right], \\
& U=1-x-\frac{1}{\left[(1+x)^{2}+y^{2}+z^{2}\right]^{3 / 2}} .
\end{aligned}
$$

Equations (13) and (14) are the governing equations of motion of deputy satellite with respect to chief satellite in the LVLH frame.

\section{Analysis of Periodic Orbits}

Since the trajectory of phase space is defined uniquely for any provided set of specified conditions, we will analyze the initial position of deputy satellite, which provides periodic orbits under different values for initial true anomalies and eccentricities of chief satellite. In this context, System (11) can be used to accomplish our goal. This system consists of secondorder nonlinear differential equations, which can be converted into system of first-order differential equations and then integrated with the Runge-Kutta fourth-order method.

The numerical integration will be developed with a step size of 0.001 during each iteration. It is important that, during simulation, true anomaly $f$ is considered as a variable. Notation $f^{0}$ stands for initial value of true anomaly. During each iteration of simulation, true anomaly $f$ varies between $f^{0}$ and $f^{0}+4 \pi$. Software MATLAB is used to perform the simulation. In this study, the periodic orbits up to ten loops are obtained for different values of $f^{0}$ and $e$, where $f^{0}$ is referred as the initial true anomaly while $e$ is the eccentricity of the orbit of chief satellite. The initial position of the deputy satellite is given as $\left(x^{0}, 0, z^{0}\right)$ and initial velocity is taken as $\left(0, y^{0}, 0\right)$. The orbital period and number of loops are denoted as $T$ and NL, respectively.

3.1. Periodic Orbits When $=0.1$. In this section, we analyze periodic orbits of deputy satellite with 1-10 loops when eccentricity $e$ of chief satellite's orbit is taken in to account as 0.1. During this analysis, the effect of initial velocity of deputy satellite in the $y$ direction and initial true anomaly of deputy satellite are considered. Thus, two different sets of initial velocities of deputy satellite $(0,0.002,0)$ and $(0,0.005,0)$ are taken into account. Also, three different values of initial true anomaly $f^{0}$ of deputy satellites are taken as $\pi / 6, \pi / 3$, and $\pi / 2$. This analysis will be investigated numerically and graphically through Figures 2-6 and Table 1. The initial position of deputy satellite and orbital period of the periodic orbits is obtained for each set of orbits with 1-10 loops. It is observed that the orbital period decreases as velocity increases for given number of loops, eccentricity, and initial true anomaly.

Figures 2(a)-2(f) show the two-dimensional view of the periodic orbits with the number of loops 1 to 6 with a given value of $x^{0}$. These orbits are obtained when the initial true anomaly and initial velocities are $\pi / 6$ and $(0,0.002,0)$, respectively.

Figures 3(a)-3(f) cover the three-dimensional view of the periodic orbits with the number of loops from 1 to 6 with a given value of $x^{0}$. These orbits are obtained when the initial true anomaly and initial velocity are $\pi / 3$ and $(0,0.002,0)$, respectively.

Figures 4(a)-4(f) show the three-dimensional view of periodic orbits with the number of loops from 5 to 10 with given value of $x^{0}$. These orbits are obtained when initial true anomaly and initial velocity are $\pi / 3$ and $(0,0.005,0)$, respectively. All the orbits are plotted in the same dimensions so that the comparative study can be possible. The comparative study of periodic orbits for different initial values of velocities depicts that shape and geometric parameters of orbits are same though the values of initial velocities are different.

Figures $5(a)-5(f)$ show the periodic orbits with the number of loops 3 to 8 with a given value of $x^{0}$. These orbits are obtained when the initial true anomaly and initial velocity are $\pi / 2$ and $(0,0.002,0)$, respectively.

The analysis of the periodic orbits for three different initial true anomalies $\pi / 6, \pi / 3$, and $\pi / 2$ are observed in Table 1 . Two different sets of initial velocities $(0,0.002,0)$ and $(0,0.005,0)$ are considered for the study when $e=0.1$. The initial position of deputy satellite and orbital period of the periodic orbits are obtained for each set of orbits with 1-10 loops. The orbital period is conserved as the velocity increases for given number of loops, eccentricity, and initial true anomaly.

The variation in initial position and period of the periodic orbits is given in Figures 6(a) and 6(b) for initial velocities $(0,0.002,0)$ and $(0,0.005,0)$, respectively. From these graphs, it can be observed that, as the number of loops in the given orbit increases, the initial position of the periodic orbit moves towards the origin (the initial position of chief satellite) irrespective of the initial value of the true anomaly. From these graphs, it can also be observed that, as number of loops in the given orbit increases, the period of the periodic orbit increases irrespective of the initial true anomaly.

3.2. Periodic Orbits When $e=0.2$. Now, we have considered the eccentricity of chief satellite's orbit as 0.2 to analyze the effect of eccentricity of chief satellite's orbit, on periodic 


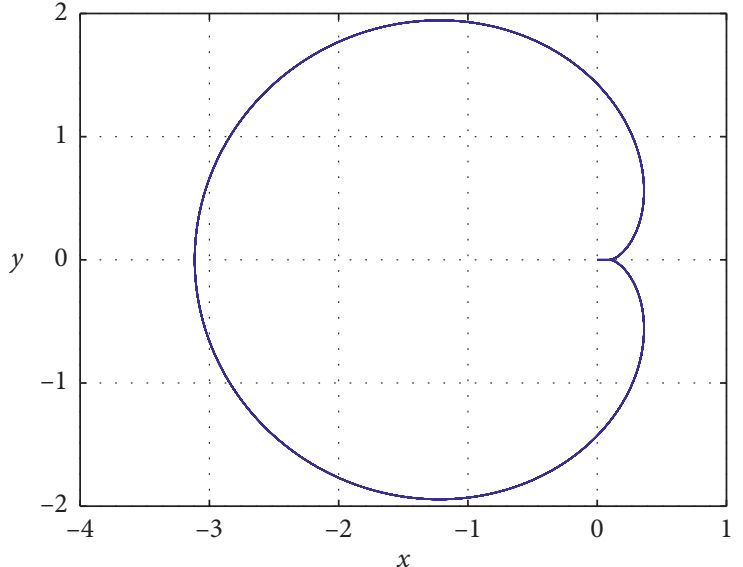

(a)

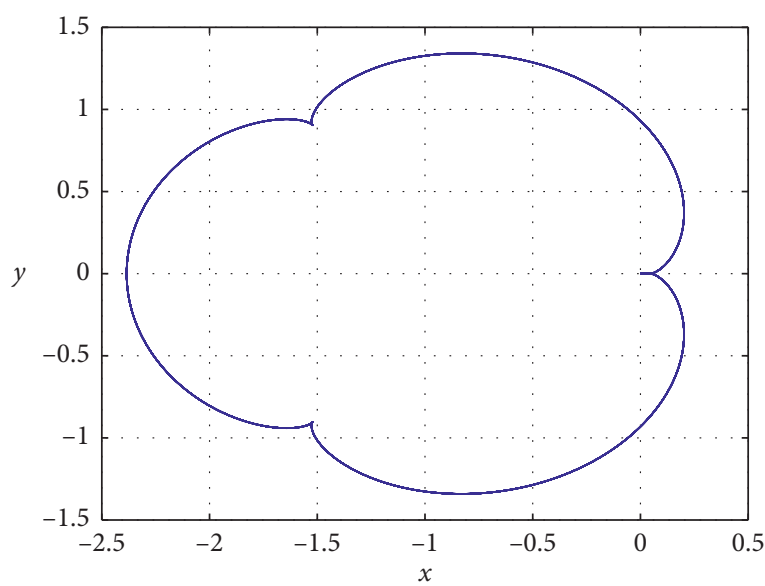

(c)

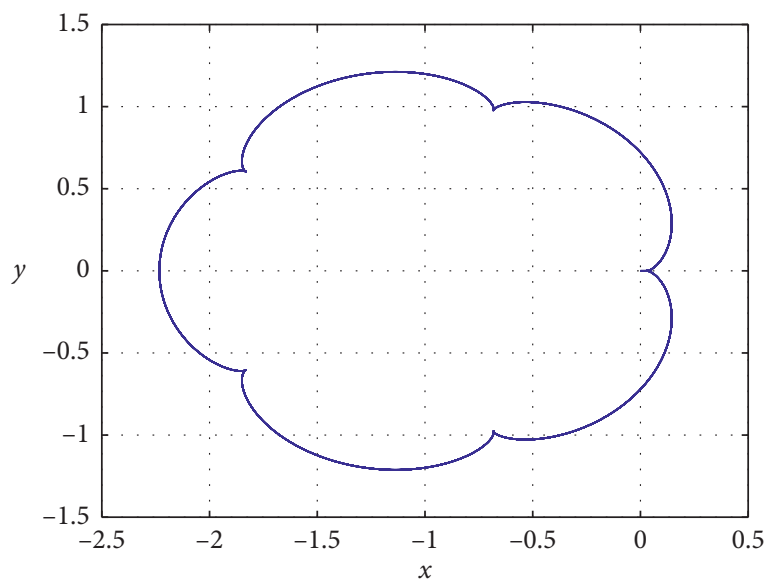

(e)

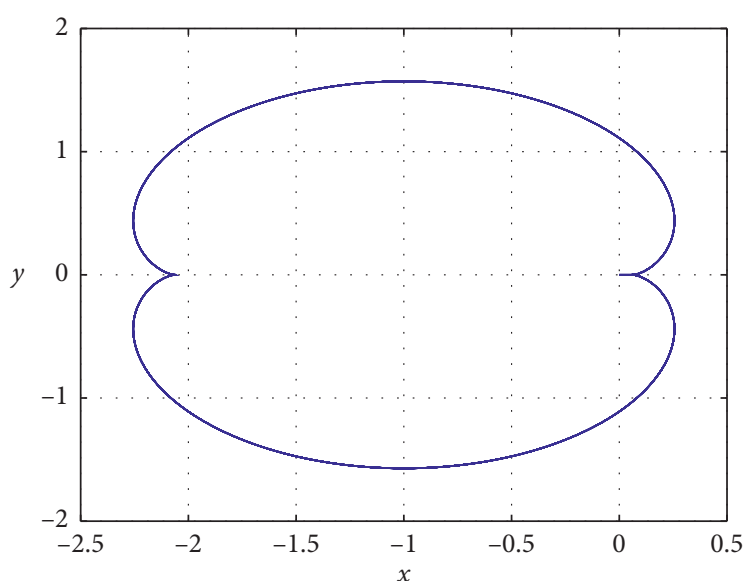

(b)

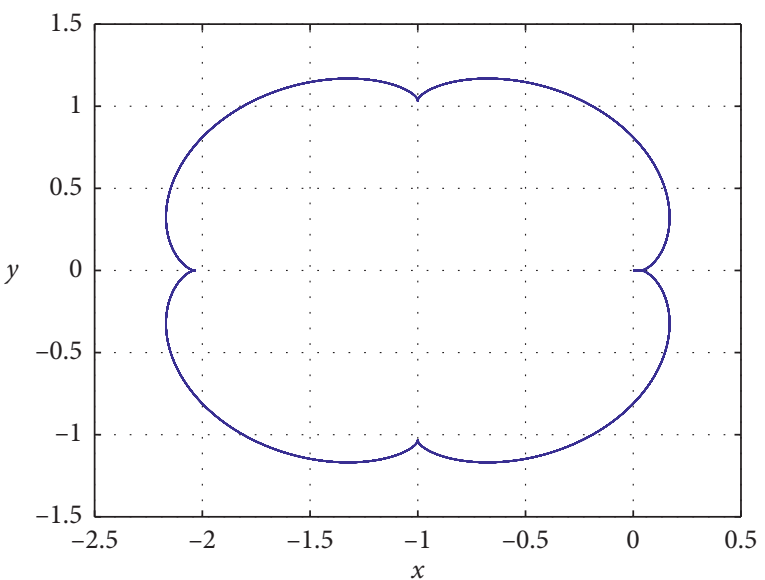

(d)

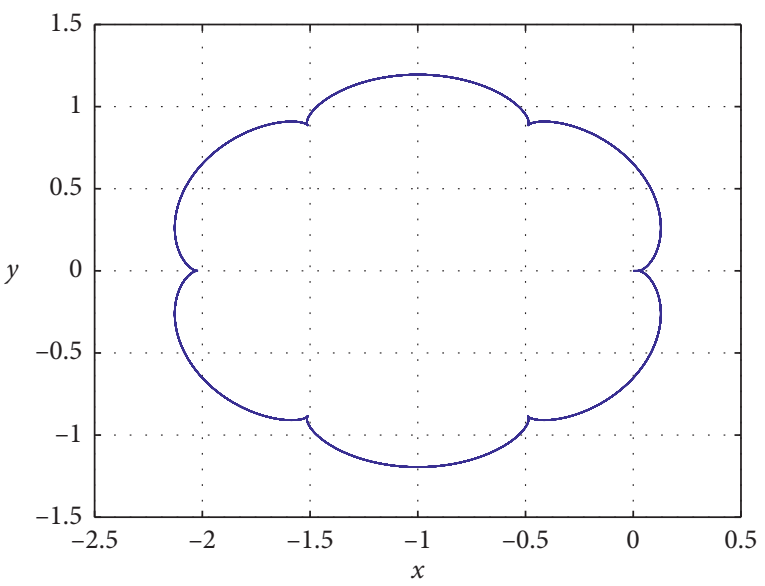

(f)

Figure 2: Periodic orbits when $e=0.1, f^{0}=\pi / 6$, and velocity $\dot{y}^{0}=0.002$. (a) Single-loop orbit, when $x^{0}=0.097990643050$. (b) Two-loop orbit, when $x^{0}=0.060941913025$. (c) Three-loop orbit, when $x^{0}=0.0442639955$. (d) Four-loop orbit, when $x^{0}=0.03469385$. (e) Five-loop orbit, when $x^{0}=0.02847325$. (f) Six-loop orbit, when $x^{0}=0.02410242$.

orbits of deputy satellite with 1-10 loops. Here, we have considered the proposed values of initial velocity of deputy satellite and initial true anomaly of deputy satellite with $e=0.1$. So that the comparative study is possible and effect of individual parameter and combination of more than one parameter can be investigated. This analysis will be 


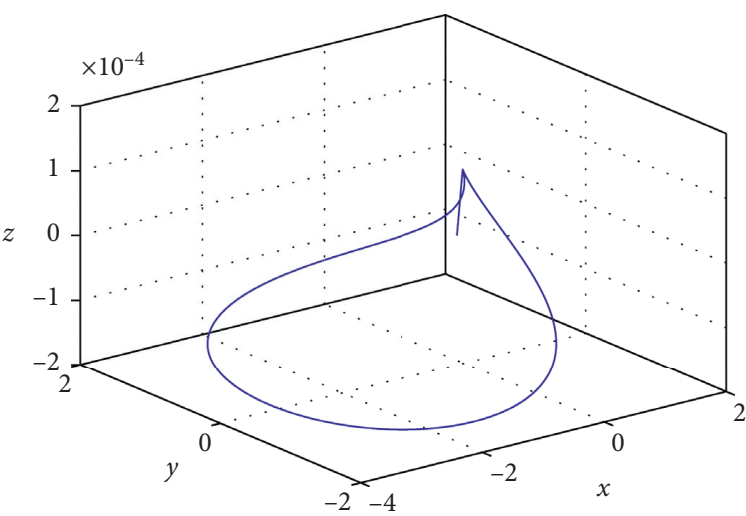

(a)

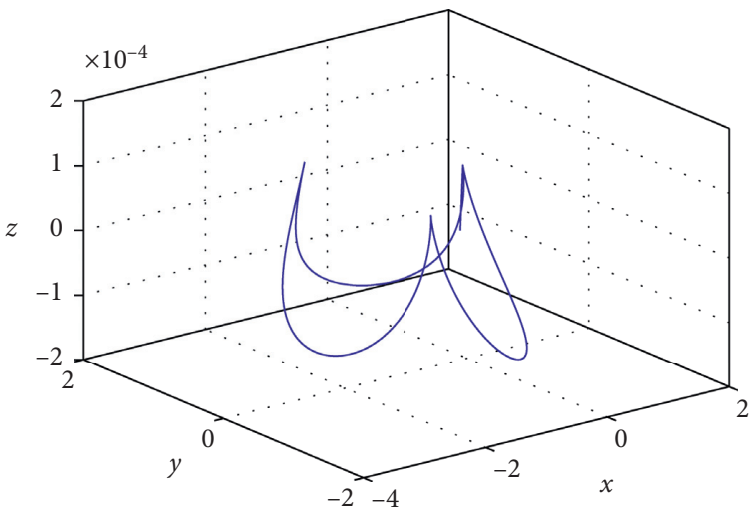

(c)

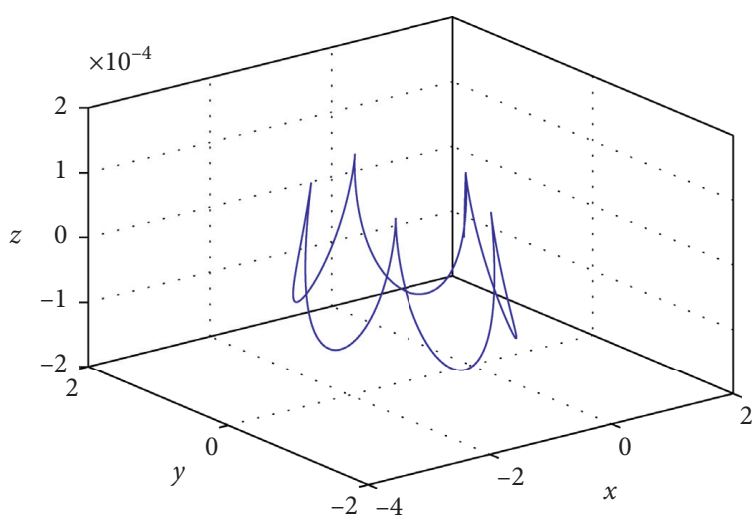

(e)

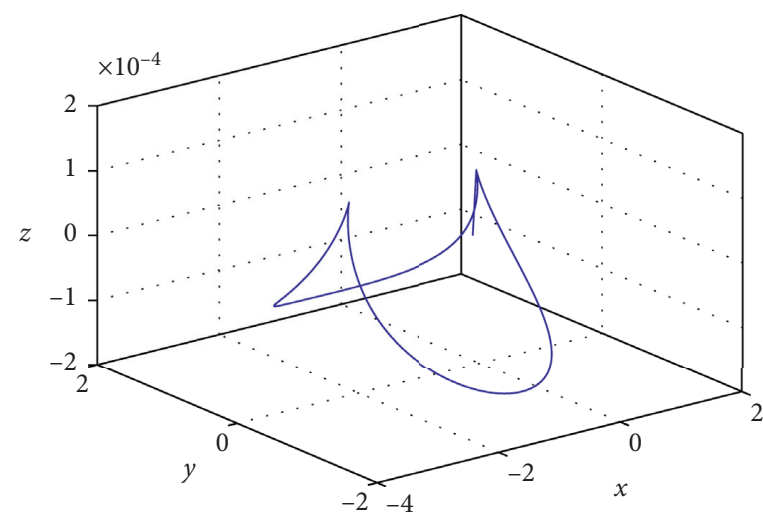

(b)

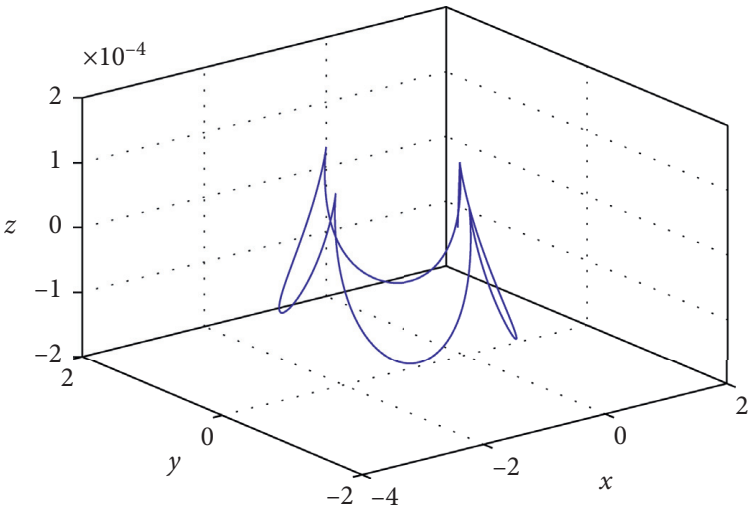

(d)

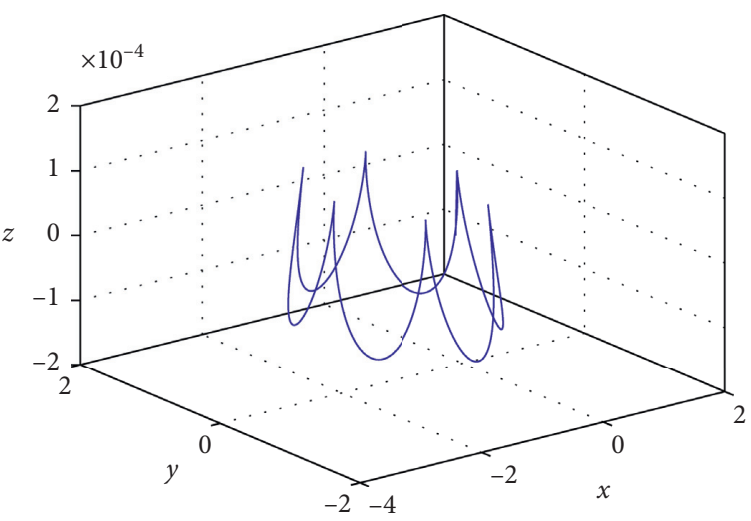

(f)

Figure 3: Periodic orbits when $e=0.1, f^{0}=\pi / 3$, and velocity $\dot{y}^{0}=0.002$. (a) Single-loop orbit, when $x^{0}=0.093525298500$. (b) Two-loop orbit, when $x^{0}=0.059057635000$. (c) Three-loop orbit, when $x^{0}=0.043092145000$. (d) Four-loop orbit, when $x^{0}=0.033847995000$. (e) Fiveloop orbit, when $x^{0}=0.027812858705$. (f) Six-loop orbit, when $x^{0}=0.0235613$.

investigated numerically and graphically through Figures $7-10$ and Table 2.

Figures $7(\mathrm{a})-7(\mathrm{f})$ show the periodic orbits with 1 to 6 number of loops with initial true anomaly and velocity of $\pi / 6$ and $(0,0.002,0), \mathrm{w} /$ respectively.

Figures 8 (a)-8(f) show the periodic orbits with 3 to 8 number of loops with an initial true anomaly and velocity of $\pi / 3$ with $(0,0.002,0)$, respectively.
Figures 9(a)-9(f) show the periodic orbits with the number of loops 5 to 10 with a given value of $x^{0}$. These orbits are obtained when the initial true anomaly and initial velocities are $\pi / 2$ and $(0,0.002,0)$, respectively.

The analysis of periodic orbits for three initial values of true anomalies $\pi / 6, \pi / 3$, and $\pi / 2$ with eccentricity $e=0.2$ is given in Table 2 . The entire study has been performed by considering two different sets of initial velocities as $(0,0.002,0)$ 


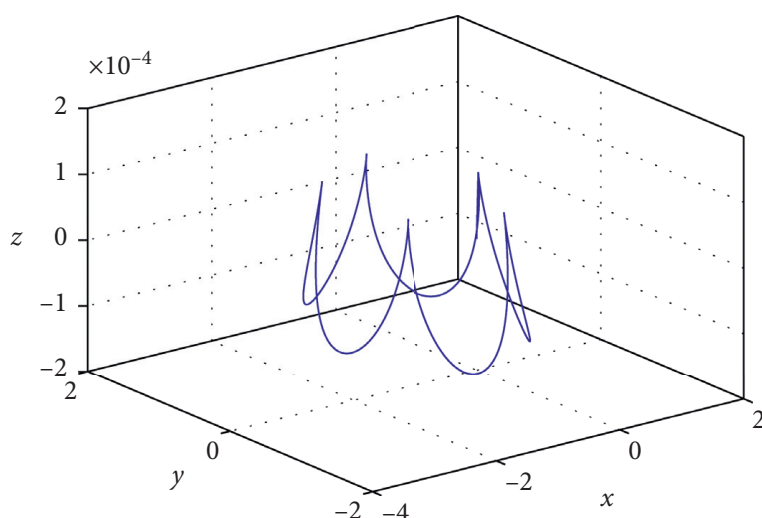

(a)

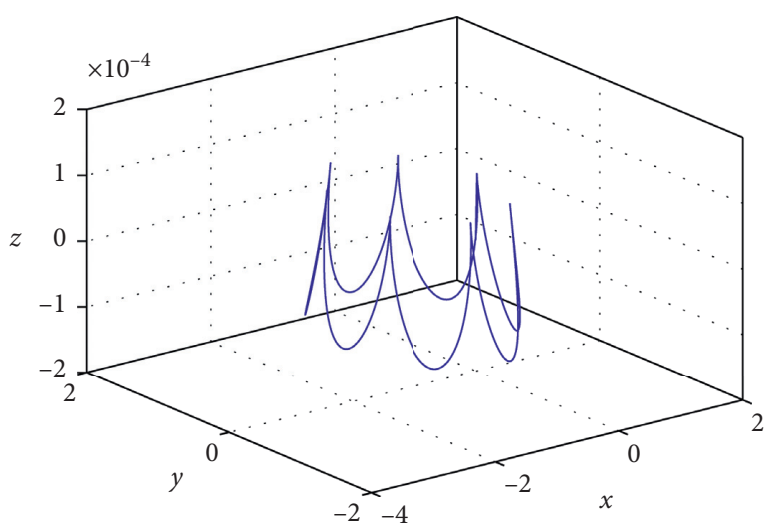

(c)

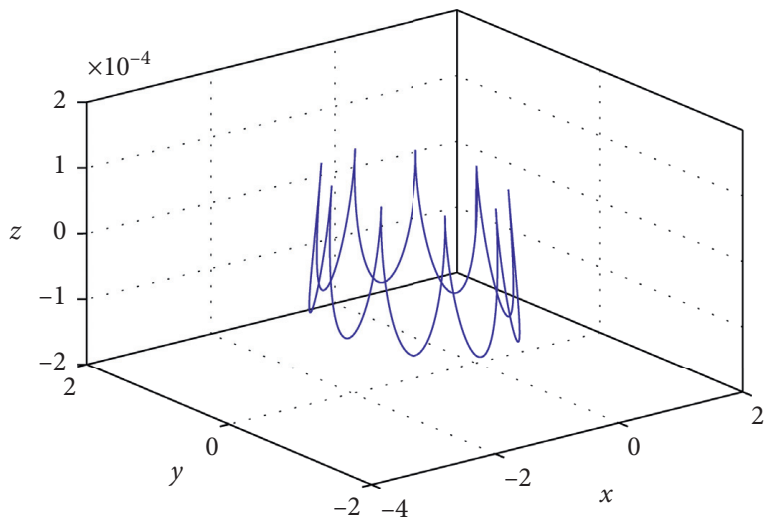

(e)

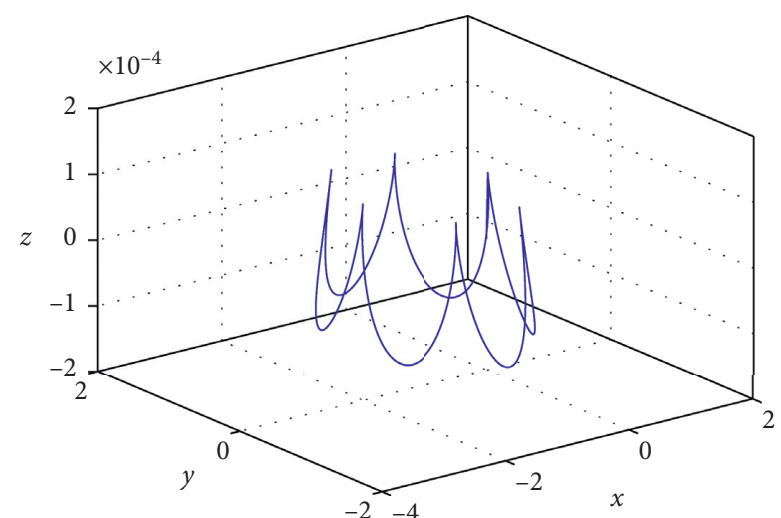

(b)

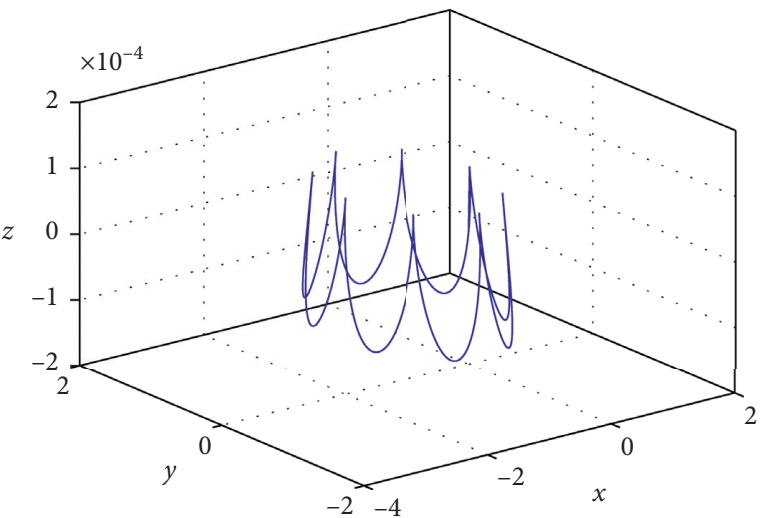

(d)

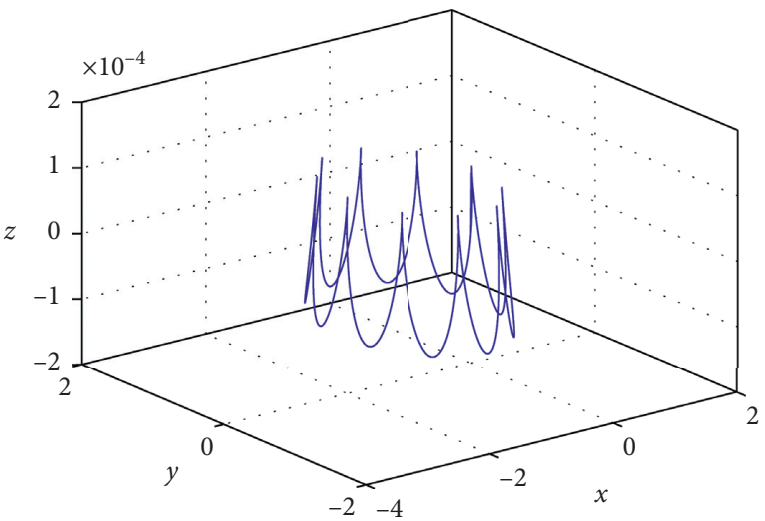

(f)

Figure 4: Periodic orbits when $e=0.1$ and $f^{0}=\pi / 3$ and velocity $\dot{y}^{0}=0.005$. (a) Five-loop orbit, when $x^{0}=0.026250375000$. (b) Six-loop orbit, when $x^{0}=0.022008125000$. (c) Seven-loop orbit, when $x^{0}=0.018857650000$. (d) Eight-loop orbit, when $x^{0}=0.016425375000$. (e) Nineloop orbit, when $x^{0}=0.01449068105$. (f) Ten-loop orbit, when $x^{0}=0.012915$.

and $(0,0.005,0)$. The initial position and period of the periodic orbits are obtained for each set of orbits with 1-10 loops.

Figures 10 (a) and 10(b) indicates the variation in the initial position and period of the periodic orbits for initial velocities $(0,0.002,0)$ and $(0,0.005,0)$, respectively. Here, eccentricity of chief satellite's orbit is considered 0.2 . From these graphs, it can be observed that, as number of loops in the given orbit increases, the initial position of the periodic orbit moves towards the origin (the initial position of chief satellite) irrespective of the initial value of true anomaly.
From these graphs, it can also be observed that, as number of loops in the given orbit increases, the period of the periodic orbit increases irrespective of the initial true anomaly.

\section{Effect of Eccentricity of Chief Satellite's Orbit}

In the previous section, we have considered that the eccentricity $e$ of chief satellite's orbit is 0.1 and 0.2 , respectively. But, in the current section, we compare the effect of variation in eccentricity of chief satellite's orbit on periodic orbits of 


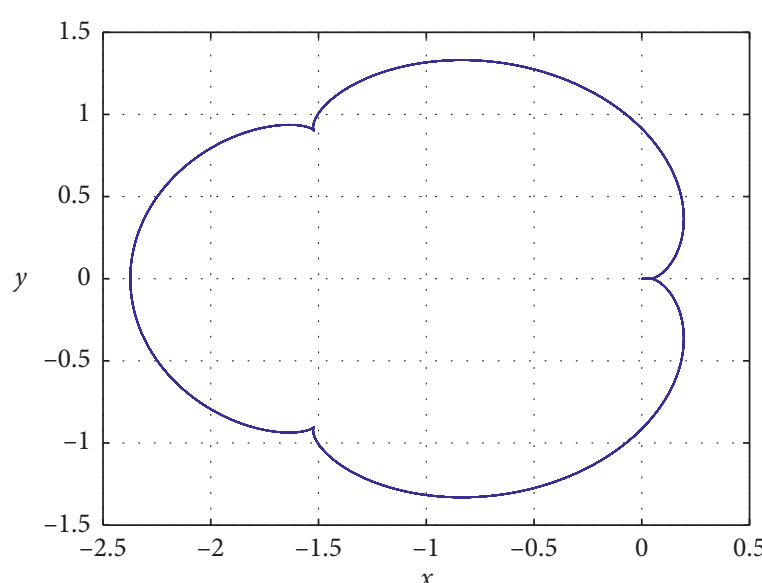

(a)

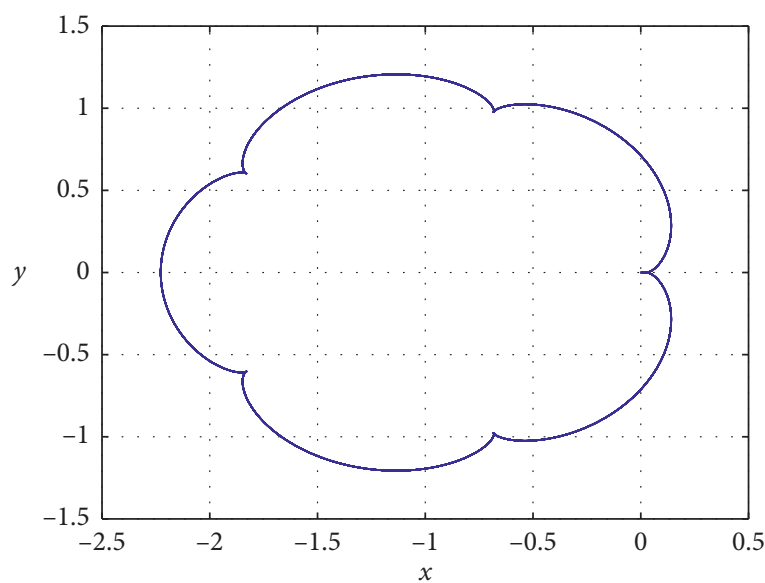

(c)

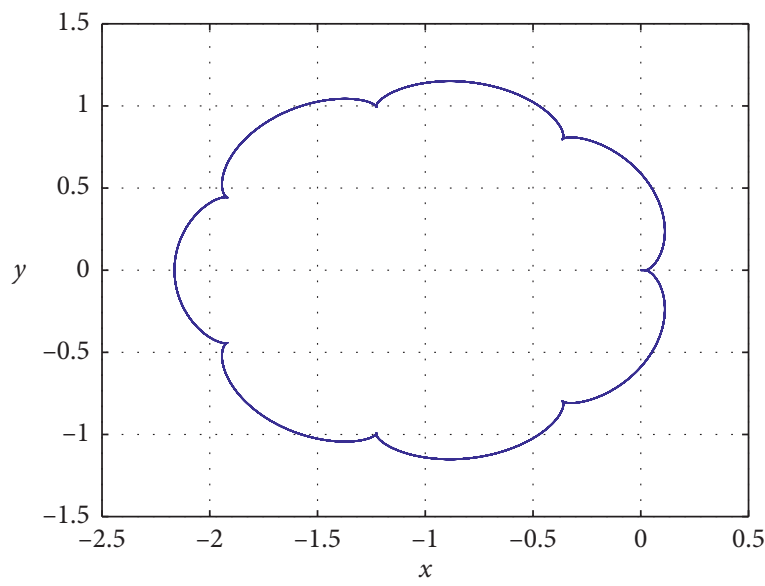

(e)

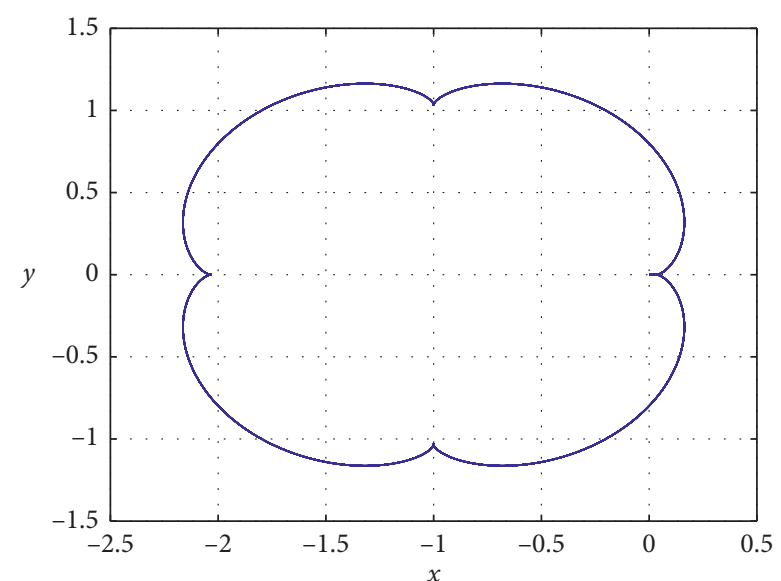

(b)

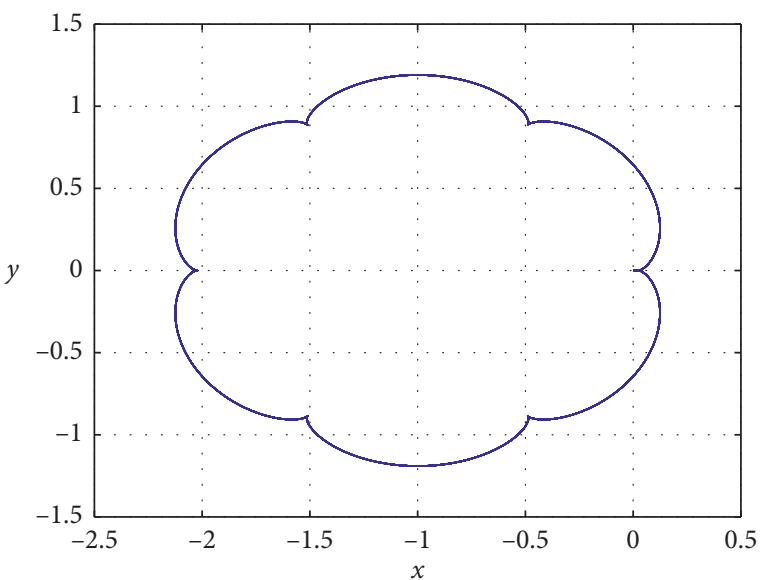

(d)

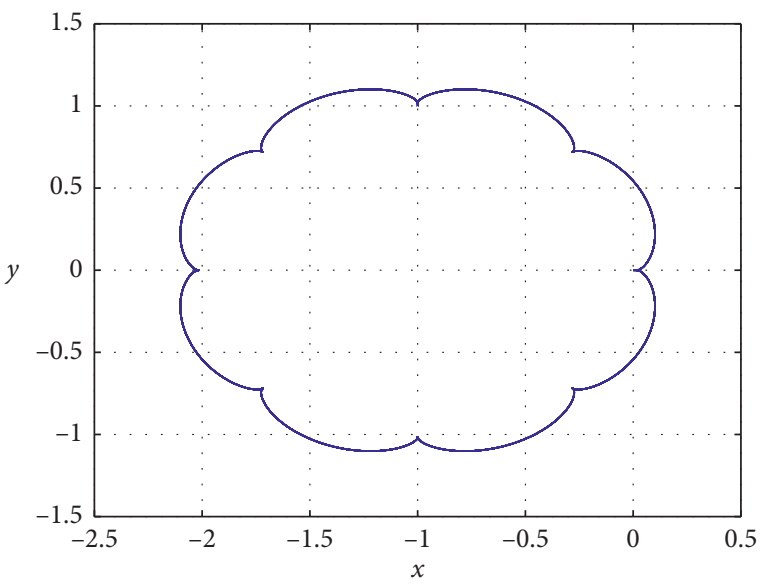

(f)

Figure 5: Periodic orbits when $e=0.1, f^{0}=\pi / 2$, and velocity $\dot{y}^{0}=0.002$. (a) Three-loop orbit, when $x^{0}=0.041398075500$. (b) Four-loop orbit, when $x^{0}=0.032620475000$. (c) Five-loop orbit, when $x^{0}=0.0268523875$. (d) Six-loop orbit, when $x^{0}=0.0227732155$. (e) Seven-loop orbit, when $x^{0}=0.0197361$. (f) Eight-loop orbit, when $x^{0}=0.017387015$.

deputy satellite with the number of loops 1-10. This effect on initial position and orbital period of periodic orbits of deputy satellite with 1-10 number of loops will be shown in Figures 11-13.
Figures 11(a) and 11(b) give the relation between the initial position and orbital period and number of loops with consideration of three different values of true anomaly as $\pi / 6, \pi / 3$, and $\pi / 2$. The velocity is taken in magnitude as $(0$, 


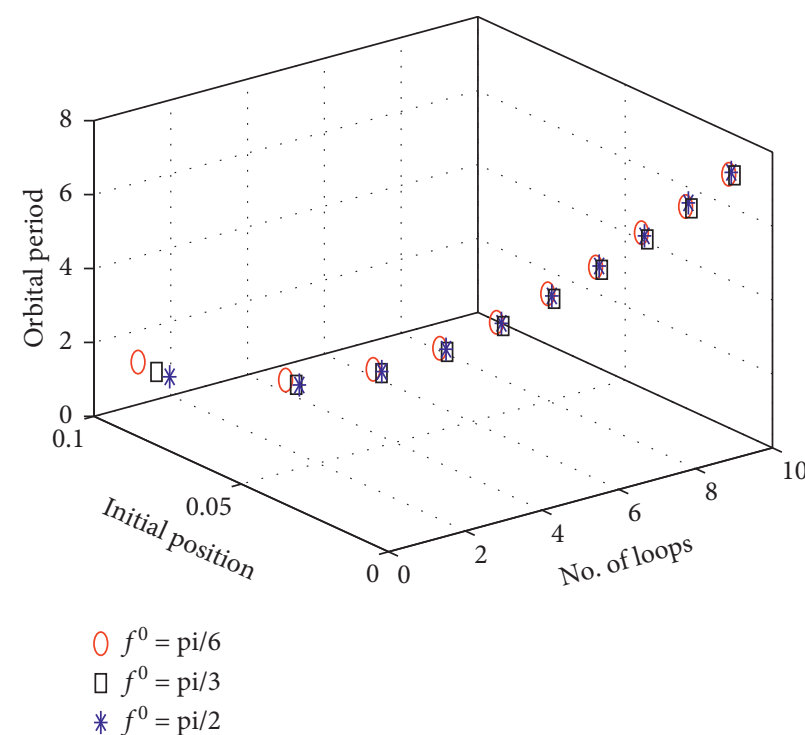

(a)

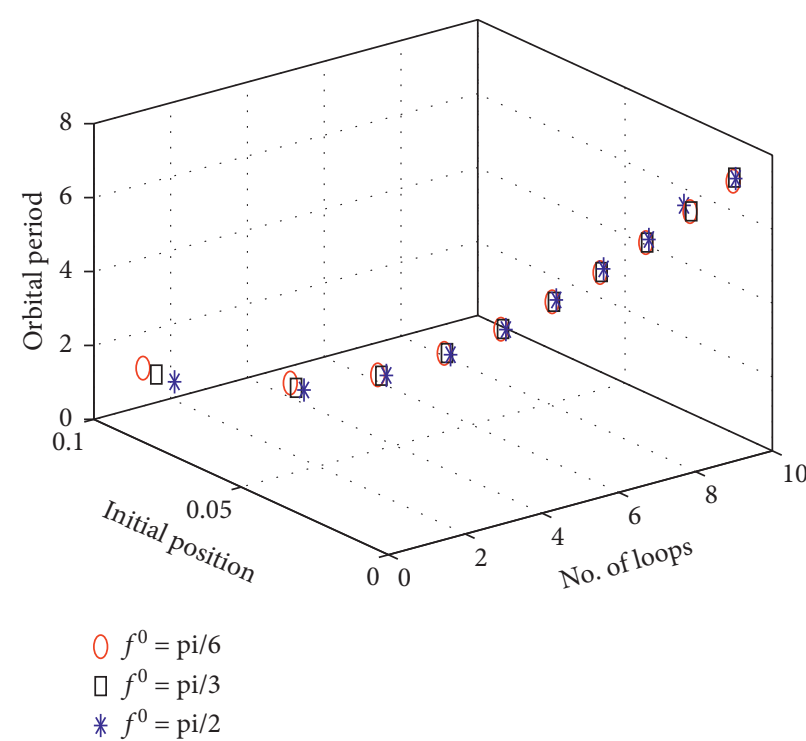

(b)

FIGURE 6: Variation in the initial position of deputy satellite and orbital period when $e=0.1$. (a) Velocity $\dot{y}^{0}=0.002$. (b) Velocity $\dot{y}^{0}=0.005$.

TABle 1: Analysis of periodic orbits with $e=0.1$.

\begin{tabular}{|c|c|c|c|c|c|}
\hline$f^{0}$ & $\dot{y}^{0}$ & $z^{0}$ & $x^{0}$ & $T$ & NL \\
\hline \multirow{10}{*}{$\pi / 6$} & \multirow{10}{*}{0.002} & \multirow{10}{*}{0.0001} & 0.097990643050 & 1.250 & 01 \\
\hline & & & 0.060941913025 & 1.885 & 02 \\
\hline & & & 0.044263995500 & 2.520 & 03 \\
\hline & & & 0.034693850000 & 3.140 & 04 \\
\hline & & & 0.028473250000 & 3.765 & 05 \\
\hline & & & 0.024102420000 & 4.400 & 06 \\
\hline & & & 0.020861979500 & 5.020 & 07 \\
\hline & & & 0.018363295500 & 5.650 & 08 \\
\hline & & & 0.016377575000 & 6.275 & 09 \\
\hline & & & 0.014761465000 & 6.900 & 10 \\
\hline \multirow{10}{*}{$\pi / 6$} & \multirow{10}{*}{0.005} & \multirow{10}{*}{0.0001} & 0.096286152500 & 1.250 & 01 \\
\hline & & & 0.059311045000 & 1.885 & 02 \\
\hline & & & 0.042667625000 & 2.520 & 03 \\
\hline & & & 0.033117650000 & 3.140 & 04 \\
\hline & & & 0.026910306500 & 3.765 & 05 \\
\hline & & & 0.022548855000 & 4.400 & 06 \\
\hline & & & 0.019315450000 & 5.020 & 07 \\
\hline & & & 0.016822145000 & 5.650 & 08 \\
\hline & & & 0.014840725000 & 6.275 & 09 \\
\hline & & & 0.013228150000 & 6.900 & 10 \\
\hline \multirow{10}{*}{$\pi / 3$} & \multirow{10}{*}{0.002} & \multirow{10}{*}{0.0001} & 0.093525298500 & 1.260 & 01 \\
\hline & & & 0.059057635000 & 1.885 & 02 \\
\hline & & & 0.043092145000 & 2.520 & 03 \\
\hline & & & 0.033847995000 & 3.140 & 04 \\
\hline & & & 0.027812858705 & 3.770 & 05 \\
\hline & & & 0.023561300000 & 4.400 & 06 \\
\hline & & & 0.020403925000 & 5.030 & 07 \\
\hline & & & 0.017966250000 & 5.655 & 08 \\
\hline & & & 0.016027250000 & 6.285 & 09 \\
\hline & & & 0.014448085000 & 6.950 & 10 \\
\hline
\end{tabular}

TABle 1: Continued.

\begin{tabular}{|c|c|c|c|c|c|}
\hline$f^{0}$ & $\dot{y}^{0}$ & $z^{0}$ & $x^{0}$ & $T$ & NL \\
\hline \multirow{10}{*}{$\pi / 3$} & \multirow{10}{*}{0.005} & \multirow{10}{*}{0.0001} & 0.091825025000 & 1.260 & 01 \\
\hline & & & 0.057428285000 & 1.885 & 02 \\
\hline & & & 0.041496687050 & 2.520 & 03 \\
\hline & & & 0.032272425000 & 3.140 & 04 \\
\hline & & & 0.026250375000 & 3.770 & 05 \\
\hline & & & 0.022008125000 & 4.400 & 06 \\
\hline & & & 0.018857650000 & 5.030 & 07 \\
\hline & & & 0.016425375000 & 5.655 & 08 \\
\hline & & & 0.014490681050 & 6.285 & 09 \\
\hline & & & 0.012915000000 & 6.950 & 10 \\
\hline \multirow{10}{*}{$\pi / 2$} & \multirow{10}{*}{0.002} & \multirow{10}{*}{0.0001} & 0.087338089500 & 1.276 & 01 \\
\hline & & & 0.056357275000 & 1.907 & 02 \\
\hline & & & 0.041398075500 & 2.540 & 03 \\
\hline & & & 0.032620475000 & 3.170 & 04 \\
\hline & & & 0.026852387500 & 3.803 & 05 \\
\hline & & & 0.022773215500 & 4.450 & 06 \\
\hline & & & 0.019736100000 & 5.070 & 07 \\
\hline & & & 0.017387015000 & 5.700 & 08 \\
\hline & & & 0.015515950000 & 6.334 & 09 \\
\hline & & & 0.013990535000 & 6.960 & 10 \\
\hline \multirow{10}{*}{$\pi / 2$} & \multirow{10}{*}{0.005} & \multirow{10}{*}{0.0001} & 0.085643905000 & 1.276 & 01 \\
\hline & & & 0.054730207500 & 1.907 & 02 \\
\hline & & & 0.039803926500 & 2.540 & 03 \\
\hline & & & 0.031045825000 & 3.170 & 04 \\
\hline & & & 0.025290650000 & 3.803 & 05 \\
\hline & & & 0.021220605000 & 4.450 & 06 \\
\hline & & & 0.018190250000 & 5.070 & 07 \\
\hline & & & 0.015846495000 & 5.700 & 08 \\
\hline & & & 0.013979735000 & 6.334 & 09 \\
\hline & & & 0.012457750000 & 6.960 & 10 \\
\hline
\end{tabular}




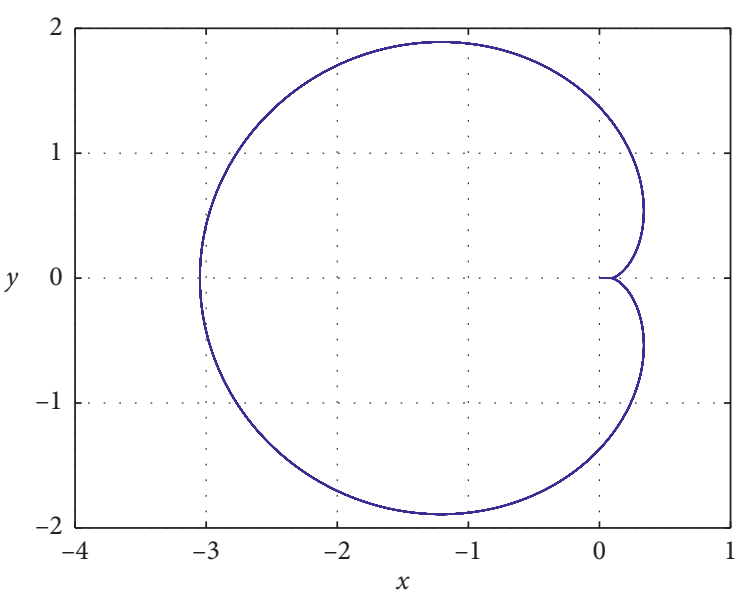

(a)

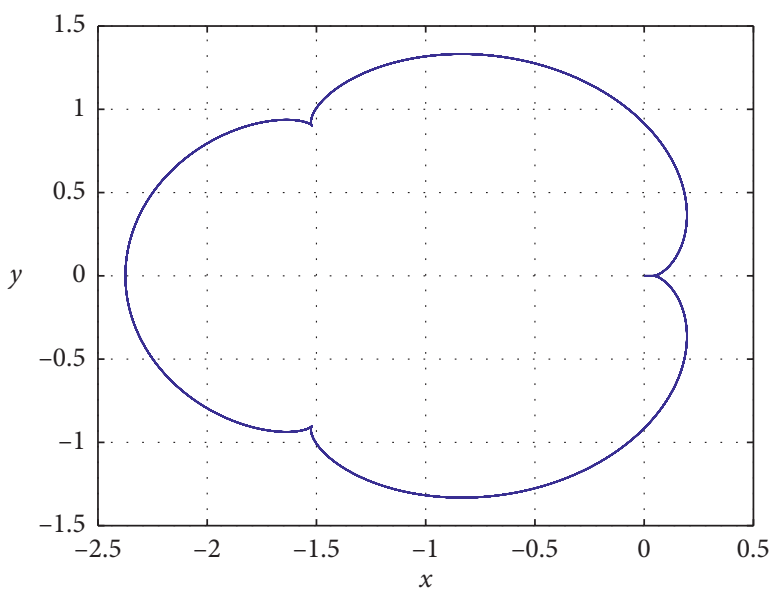

(c)

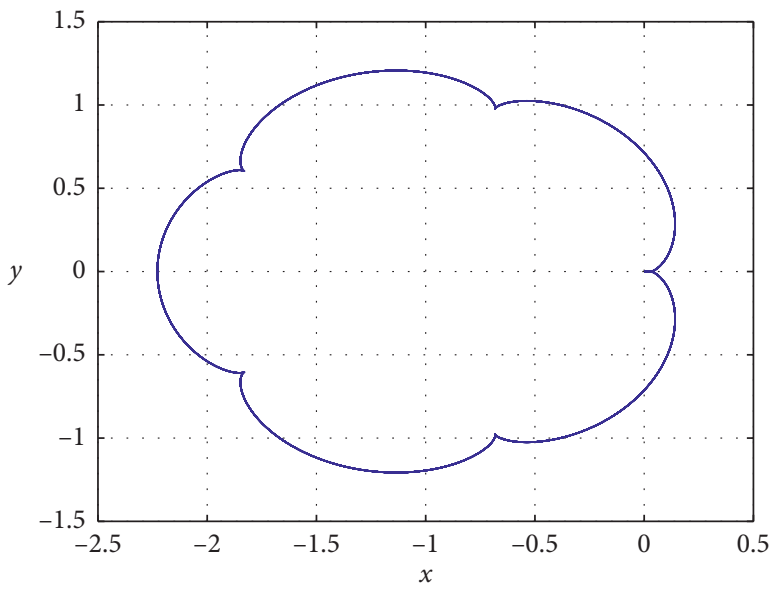

(e)

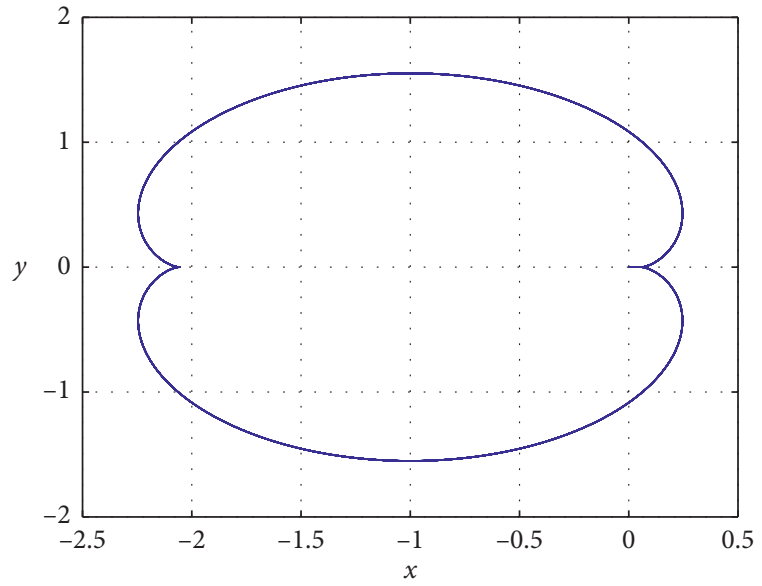

(b)

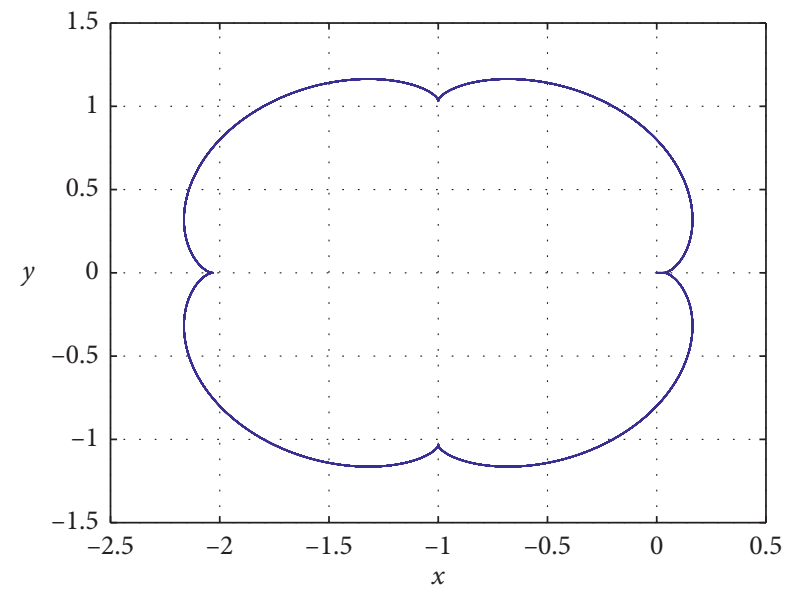

(d)

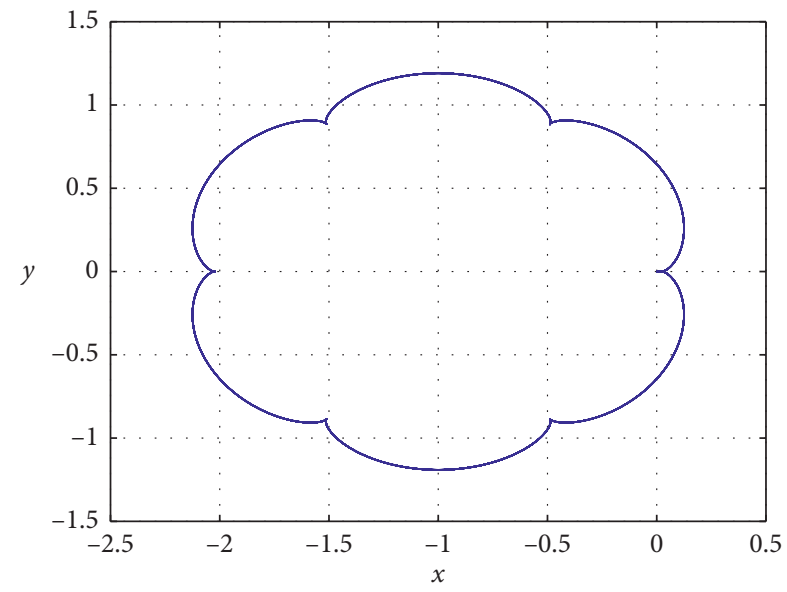

(f)

Figure 7: Periodic orbits when $e=0.2, f^{0}=\pi / 6$, and velocity $\dot{y}^{0}=0.002$. (a) One-loop orbit, when $x^{0}=0.088265415$. (b) Two-loop orbit, when $x^{0}=0.0567688625$. (c) Three-loop orbit, when $x^{0}=0.041657425$. (d) Four-loop orbit, when $x^{0}=0.032808755$. (e) Five-loop orbit, when $x^{0}=0.026999870500$. (f) Six-loop orbit, when $x^{0}=0.022894322500$.

$0.002,0)$. It has been observed from the Figure 11(a) for single-loop orbit that, as the true anomaly increases, the initial position of the periodic orbit moves towards zero. Thus, it has been observed from the comparative study that, in both the cases of initial velocities and eccentricities, as true anomaly increases, the initial position of periodic orbit moves towards zero. Also, it can be seen that, as we shift towards 1- to 10-loop periodic orbit, the difference in the initial position due to the true anomaly decreases. Thus, initial locations of periodic orbits for three different values of 


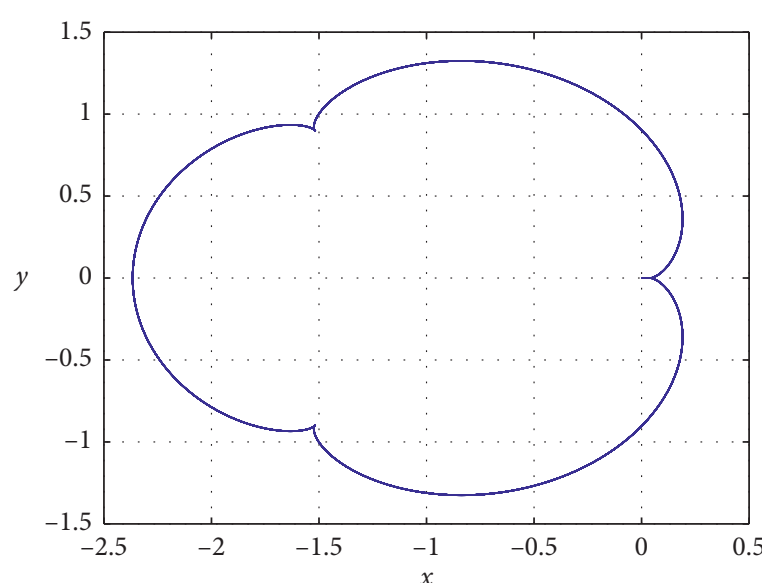

(a)

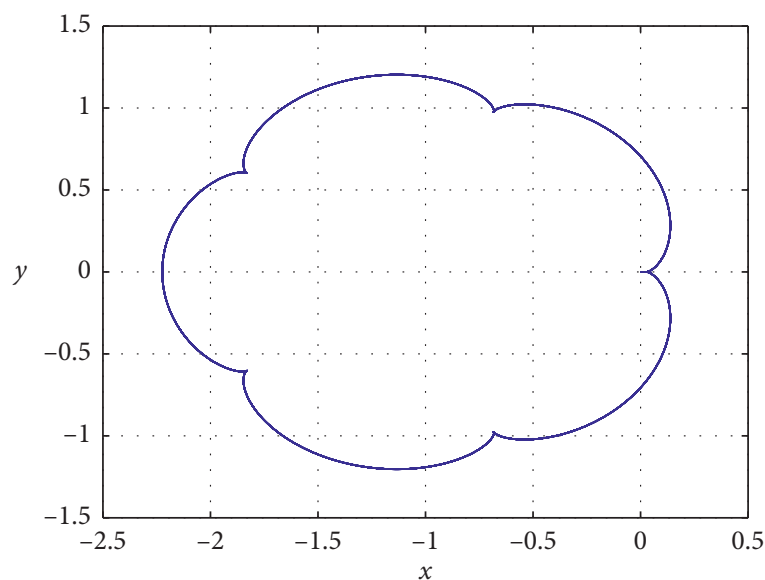

(c)

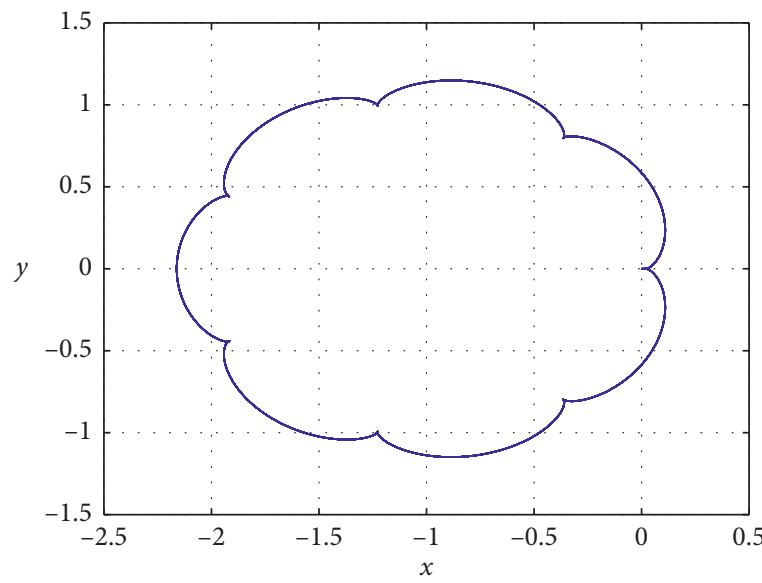

(e)

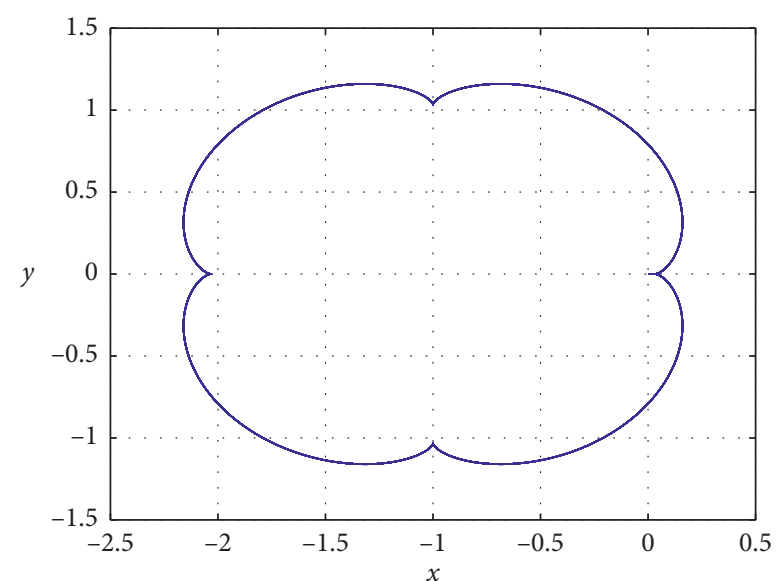

(b)

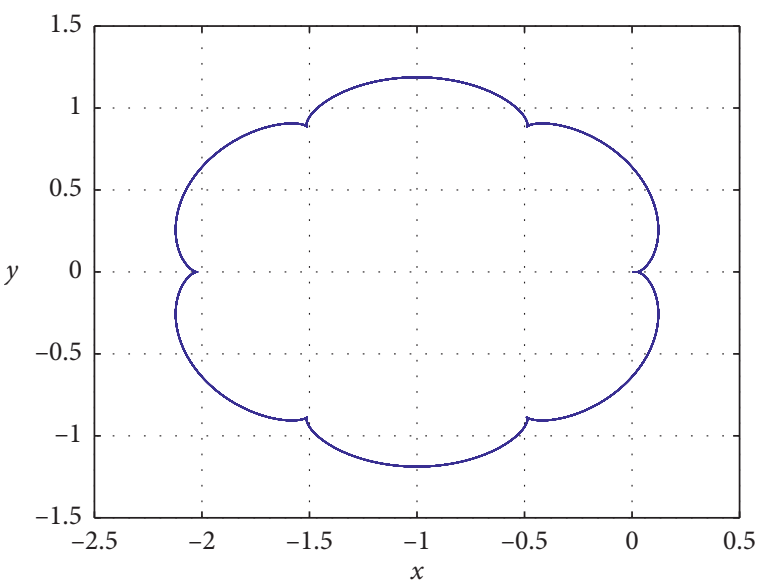

(d)

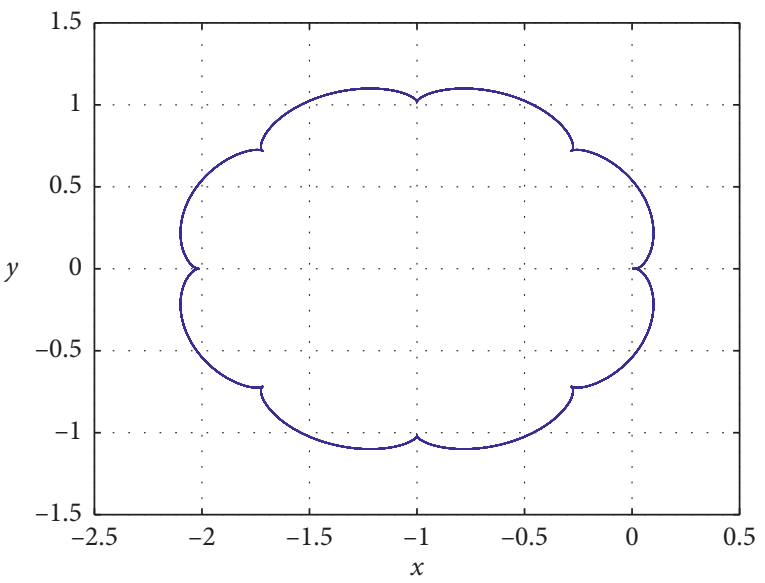

(f)

Figure 8: Periodic orbits when $e=0.2, f^{0}=\pi / 3$ and velocity $\dot{y}^{0}=0.002$. (a) Three loops orbit, when $x^{0}=0.0396983975$. (b) Four loops orbit, when $x^{0}=0.031383115$. (c) Five loops orbit, when $x^{0}=0.0258816925$. (d) Six loops orbit, when $x^{0}=0.02197541025$. (e) Seven loops orbit, when $x^{0}=0.019059245000$. (f) Eight loops orbit, when $x^{0}=0.016799437500$.

true anomaly come closer to each other as the number of loops increases from 1 to 10 . Variation in periods of periodic orbits with respect to the number of loops for three different values of true anomaly as $\pi / 6, \pi / 3$, and $\pi / 2$ is shown in Figure 11 (b) with a velocity $(0,0.002,0)$. It has been seen in both cases of initial velocities and eccentricities that orbital period increases with the increasing number of loops.

Figures 12(a) and 12(b) give the relation between the initial position and orbital period and number of loops with consideration of three different values of true anomaly as 


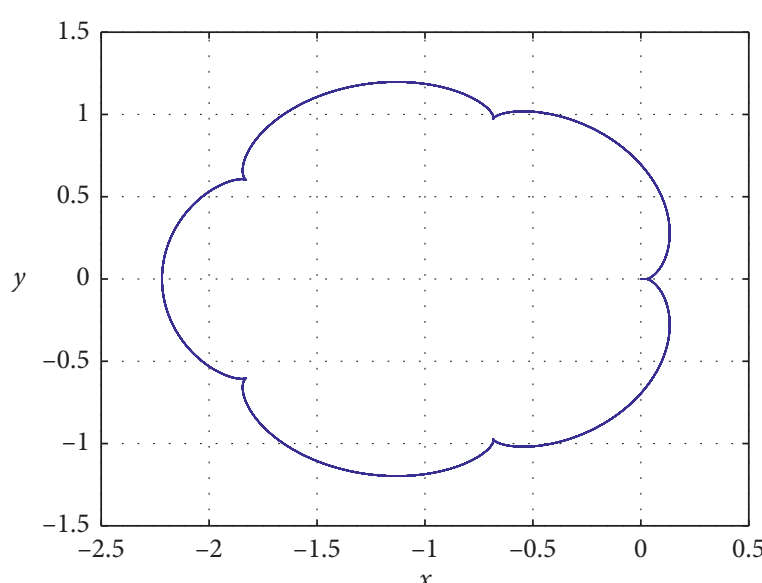

(a)

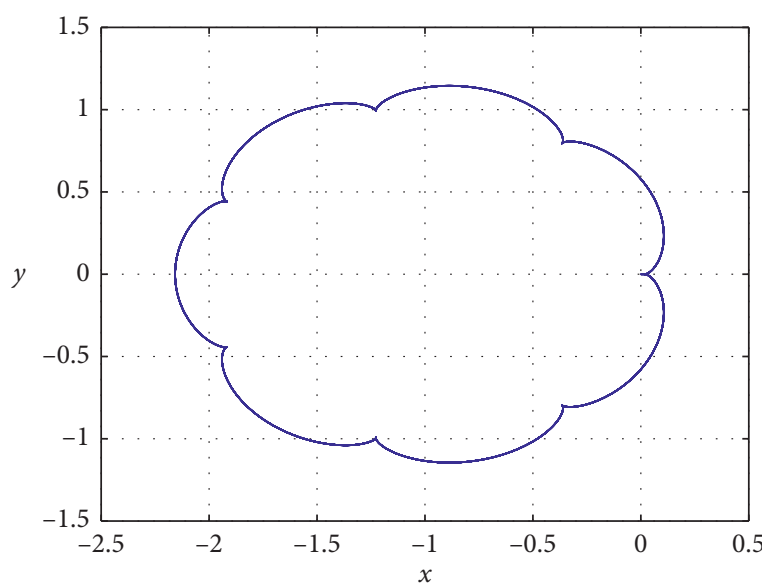

(c)

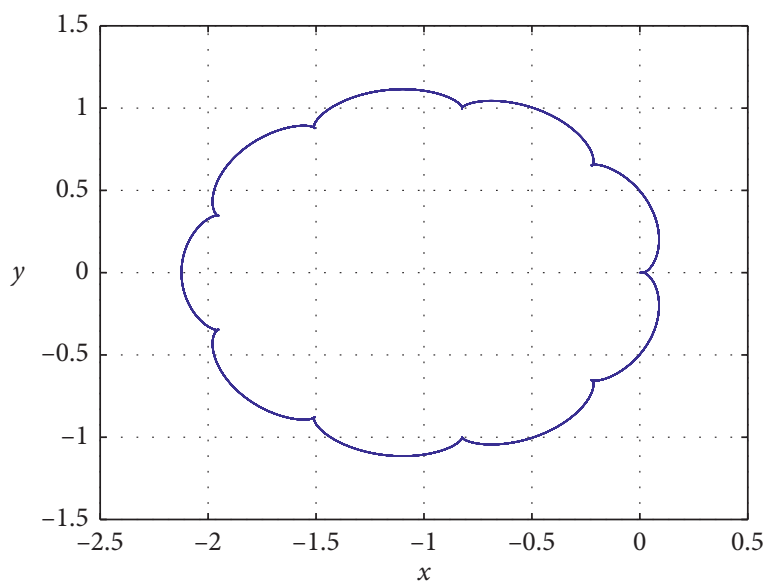

(e)

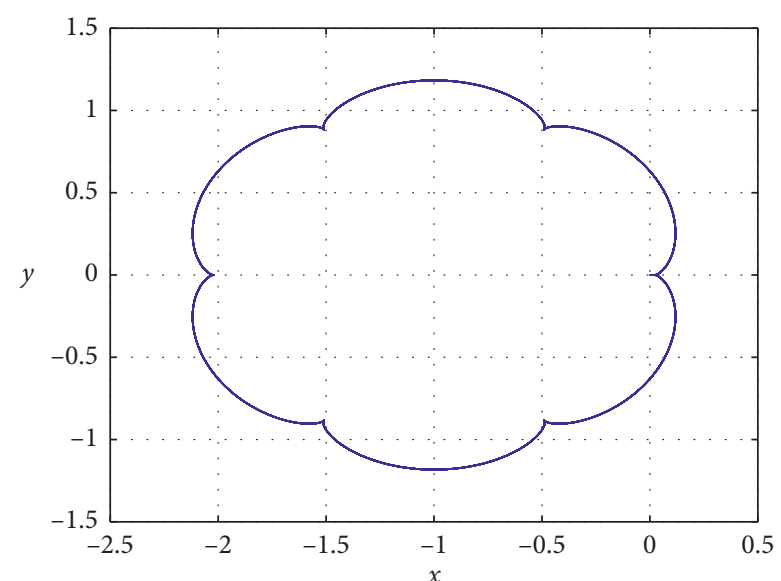

(b)

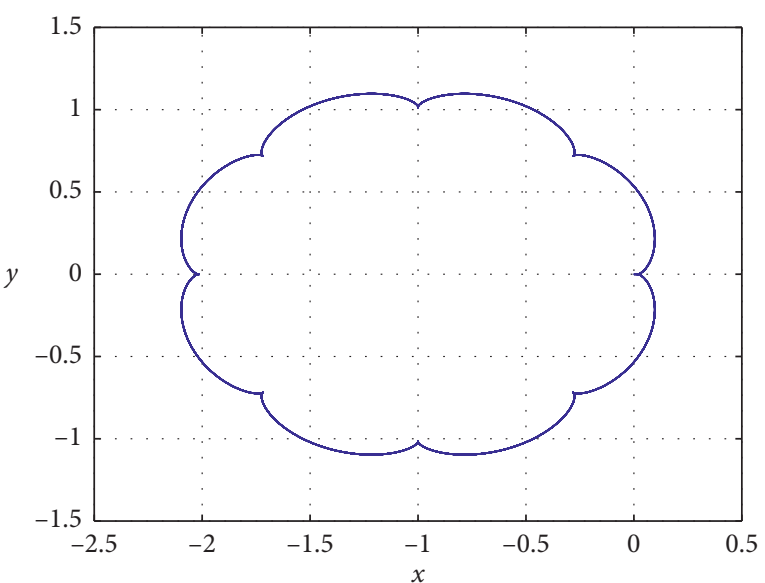

(d)

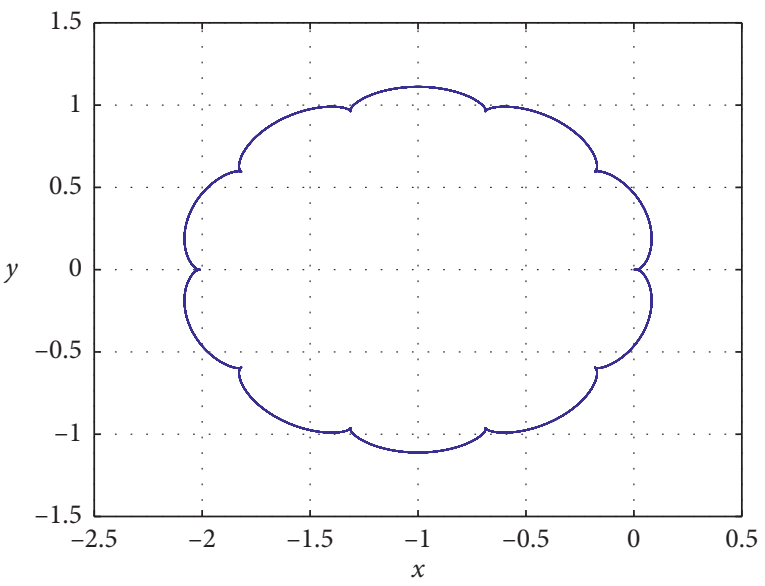

(f)

Figure 9: Periodic orbits when $e=0.2, f^{0}=\pi / 2$, and velocity $\dot{y}^{0}=0.002$. (a) Five-loop orbit, when $x^{0}=0.024156265$. (b) Six-loop orbit, when $x^{0}=0.020553885$. (c) Seven-loop orbit, when $x^{0}=0.01785125$. (d) Eight-loop orbit, when $x^{0}=0.0157495245$. (e) Nine-loop orbit, when $x^{0}=0.029191930500$. (f) Ten-loop orbit, when $x^{0}=0.027620026500$.

$\pi / 6, \pi / 3$, and $\pi / 2$. The velocity is taken in magnitude as $(0$, $0.005,0)$. It has been observed from the Figure 12(a) for single-loop orbit that, as the true anomaly increases, the initial position of periodic orbit moves towards zero. Thus, it has been observed from the comparative study that, in both the cases of initial velocities and eccentricities, as true anomaly increases, the initial position of periodic orbit moves towards zero. Also, it can be seen that, as we shift towards 1 - to 10-loop periodic orbit, the difference in the initial position due to the true anomaly decreases. Thus, the 

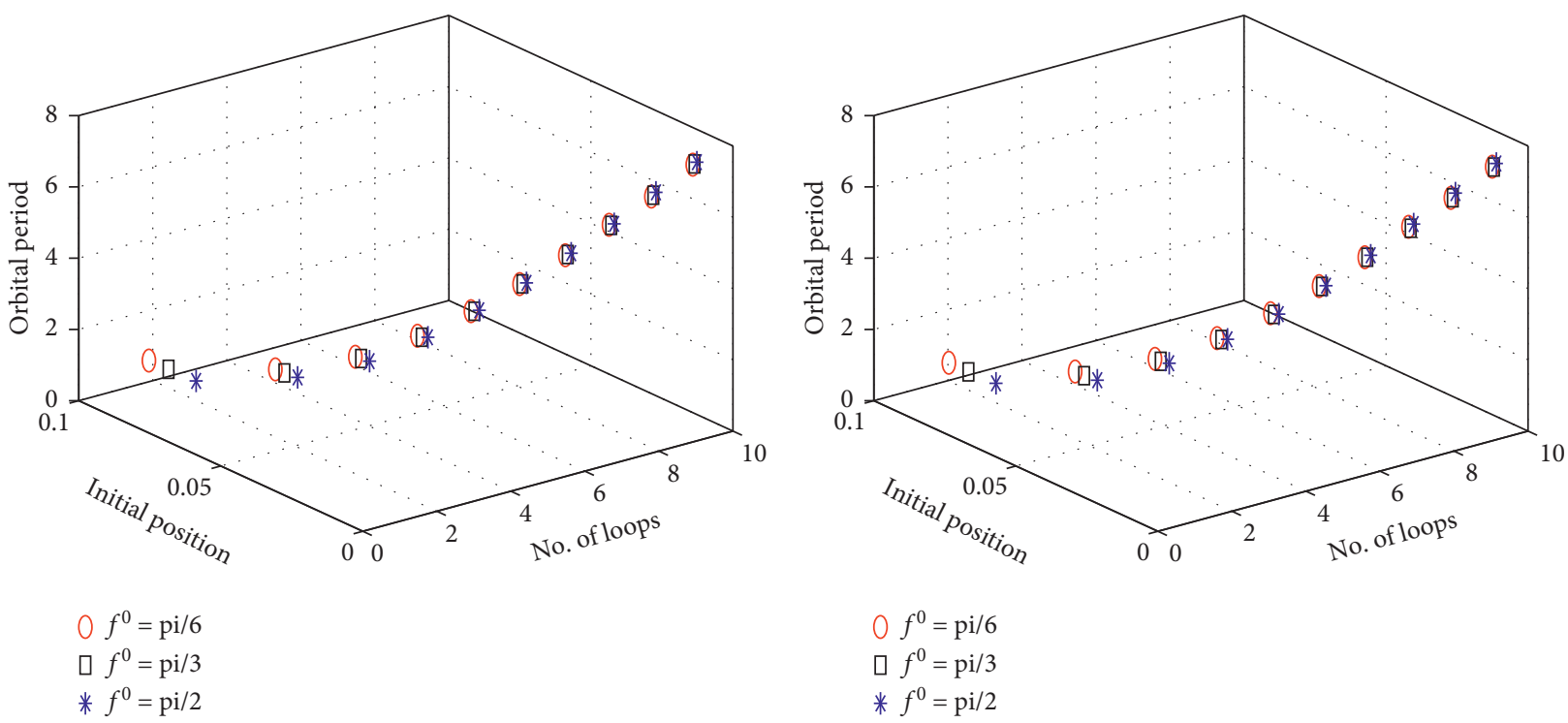

$$
\begin{aligned}
& 0 f^{0}=\mathrm{pi} / 6 \\
& \square f^{0}=\mathrm{pi} / 3 \\
& * f^{0}=\mathrm{pi} / 2
\end{aligned}
$$

(a)

(b)

FIgURE 10: Variation in the initial position of deputy satellite and orbital period when $e=0.2$. (a) Velocity $\dot{y}^{0}=0.002$. (b) Velocity

\begin{tabular}{|c|c|c|c|c|c|}
\hline$f^{0}$ & $\dot{y}^{0}$ & $z^{0}$ & $x^{0}$ & $T$ & $\mathrm{NL}$ \\
\hline \multirow{10}{*}{$\pi / 6$} & \multirow{10}{*}{0.002} & \multirow{10}{*}{0.0001} & 0.088265415000 & 1.272 & 01 \\
\hline & & & 0.056768862500 & 1.910 & 02 \\
\hline & & & 0.041657425000 & 2.533 & 03 \\
\hline & & & 0.032808755000 & 3.165 & 04 \\
\hline & & & 0.026999870500 & 3.797 & 05 \\
\hline & & & 0.022894322500 & 4.450 & 06 \\
\hline & & & 0.019838765000 & 5.062 & 07 \\
\hline & & & 0.017476085000 & 5.700 & 08 \\
\hline & & & 0.015594612750 & 6.325 & 09 \\
\hline & & & 0.014060927500 & 6.956 & 10 \\
\hline \multirow{10}{*}{$\pi / 6$} & \multirow{10}{*}{0.005} & \multirow{10}{*}{0.0001} & 0.086570301500 & 1.272 & 01 \\
\hline & & & 0.055141436550 & 1.910 & 02 \\
\hline & & & 0.040063075000 & 2.533 & 03 \\
\hline & & & 0.031233975000 & 3.165 & 04 \\
\hline & & & 0.025437996500 & 3.797 & 05 \\
\hline & & & 0.021341635000 & 4.450 & 06 \\
\hline & & & 0.018292920100 & 5.062 & 07 \\
\hline & & & 0.015935540000 & 5.700 & 08 \\
\hline & & & 0.014058295500 & 6.325 & 09 \\
\hline & & & 0.012528082500 & 6.956 & 10 \\
\hline \multirow{10}{*}{$\pi / 3$} & \multirow{10}{*}{0.002} & \multirow{10}{*}{0.0001} & 0.081430725000 & 1.293 & 01 \\
\hline & & & 0.053676025000 & 1.926 & 02 \\
\hline & & & 0.039698397500 & 2.600 & 03 \\
\hline & & & 0.031383115000 & 3.200 & 04 \\
\hline & & & 0.025881692500 & 3.837 & 05 \\
\hline & & & 0.021975410250 & 4.474 & 06 \\
\hline & & & 0.019059245000 & 5.115 & 07 \\
\hline & & & 0.016799437500 & 5.748 & 08 \\
\hline & & & 0.014996967500 & 6.386 & 09 \\
\hline & & & 0.013525826550 & 7.025 & 10 \\
\hline
\end{tabular}
$\dot{y}^{0}=0.005$.

TABLE 2: Analysis of periodic orbits with $e=0.2$.

\begin{tabular}{|c|c|c|c|c|c|}
\hline$f^{0}$ & $\dot{y}^{0}$ & $z^{0}$ & $x^{0}$ & $T$ & NL \\
\hline \multirow{10}{*}{$\pi / 3$} & \multirow{10}{*}{0.005} & \multirow{10}{*}{0.0001} & 0.079742645000 & 1.293 & 01 \\
\hline & & & 0.052051320000 & 1.926 & 02 \\
\hline & & & 0.038105645000 & 2.600 & 03 \\
\hline & & & 0.029809435000 & 3.200 & 04 \\
\hline & & & 0.024320665000 & 3.837 & 05 \\
\hline & & & 0.020423376500 & 4.474 & 06 \\
\hline & & & 0.017513965000 & 5.115 & 07 \\
\hline & & & 0.015259385000 & 5.748 & 08 \\
\hline & & & 0.013461085000 & 6.386 & 09 \\
\hline & & & 0.011993350000 & 7.025 & 10 \\
\hline \multirow{10}{*}{$\pi / 2$} & \multirow{10}{*}{0.002} & \multirow{10}{*}{0.0001} & 0.071713530000 & 1.328 & 01 \\
\hline & & & 0.049027550000 & 1.968 & 02 \\
\hline & & & 0.036708365000 & 2.612 & 03 \\
\hline & & & 0.029191930500 & 3.257 & 04 \\
\hline & & & 0.024156265000 & 3.903 & 05 \\
\hline & & & 0.020553885000 & 4.550 & 06 \\
\hline & & & 0.017851250000 & 5.195 & 07 \\
\hline & & & 0.015749524500 & 5.843 & 08 \\
\hline & & & 0.014068710500 & 6.488 & 09 \\
\hline & & & 0.012694067500 & 7.135 & 10 \\
\hline \multirow{10}{*}{$\pi / 2$} & \multirow{10}{*}{0.005} & \multirow{10}{*}{0.0001} & 0.070036225000 & 1.328 & 01 \\
\hline & & & 0.047407175000 & 1.968 & 02 \\
\hline & & & 0.035118165000 & 2.612 & 03 \\
\hline & & & 0.027620026500 & 3.257 & 04 \\
\hline & & & 0.022596595500 & 3.903 & 05 \\
\hline & & & 0.019002955000 & 4.550 & 06 \\
\hline & & & 0.016306865000 & 5.195 & 07 \\
\hline & & & 0.014210250000 & 5.843 & 08 \\
\hline & & & 0.012533505000 & 6.488 & 09 \\
\hline & & & 0.011162205000 & 7.135 & 10 \\
\hline
\end{tabular}

TABLE 2: Continued. 

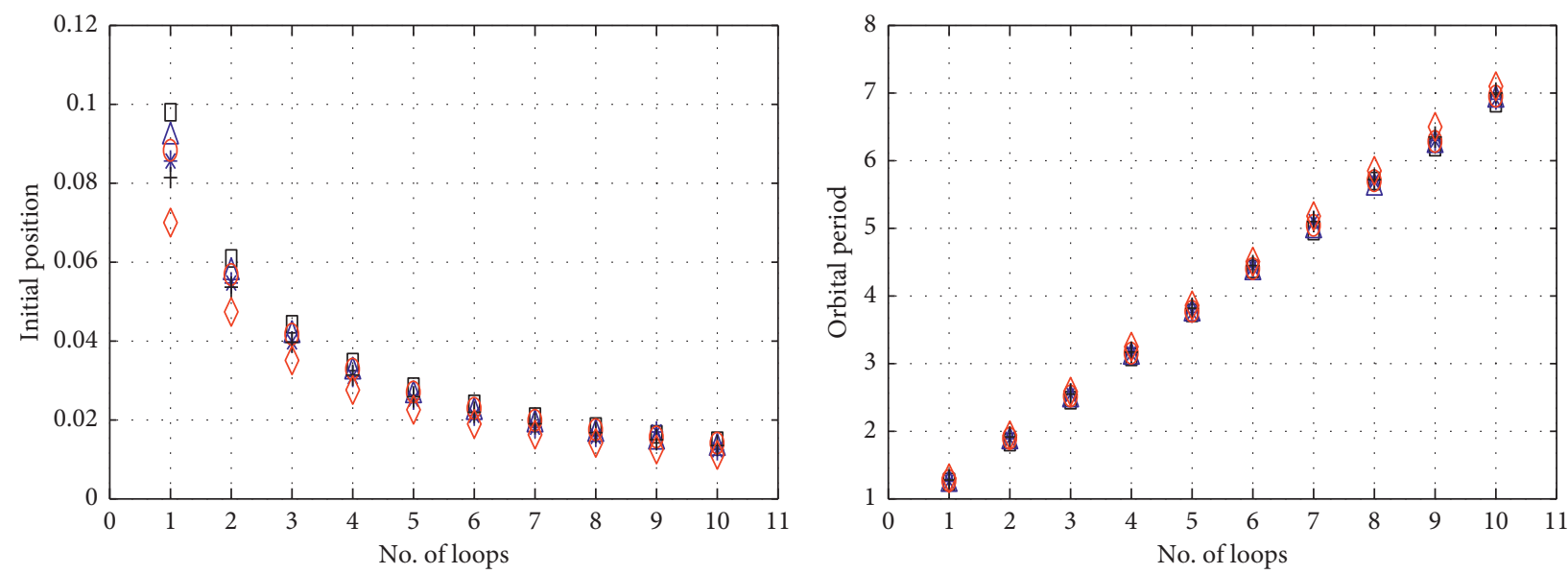
( $f^{0}=\mathrm{pi} / 6, e=0.1$
$\Delta f^{0}=\mathrm{pi} / 3, e=0.1$
* $f^{0}=\mathrm{pi} / 2, e=0.1$

\begin{abstract}
$f^{0}=\mathrm{pi} / 6, e=0.2$
$+f^{0}=\mathrm{pi} / 3, e=0.2$

$\Delta f^{0}=\mathrm{pi} / 2, e=0.2$
\end{abstract}

(a)
ㄱ $f^{0}=\mathrm{pi} / 6, e=0.1$
O $f^{0}=\mathrm{pi} / 6, e=0.2$
$\Delta f^{0}=\mathrm{pi} / 3, e=0.1$
$+f^{0}=\mathrm{pi} / 3, e=0.2$
* $f^{0}=\mathrm{pi} / 2, e=0.1$
$\diamond f^{0}=\mathrm{pi} / 2, e=0.2$

(b)

Figure 11: Variation in (a) the initial position of periodic orbits and (b) orbital period when $\dot{y}^{0}=0.002$.

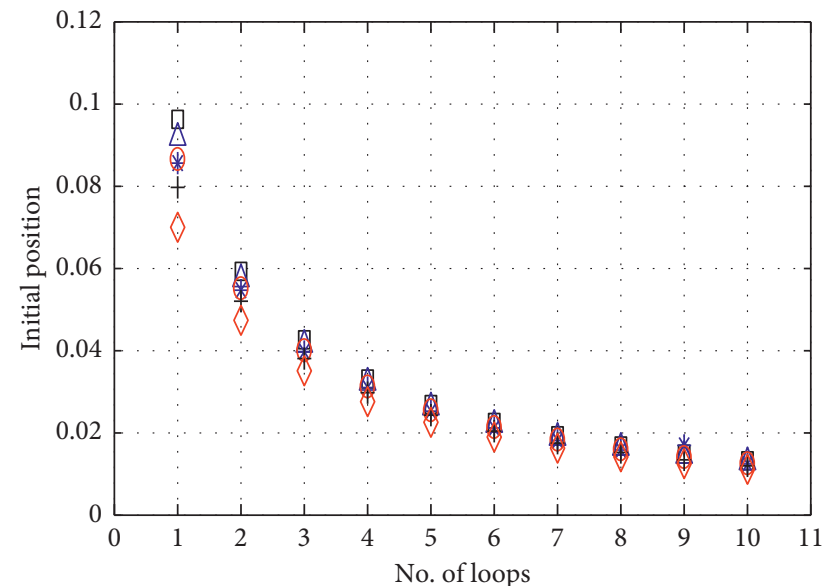
$\square f^{0}=\mathrm{pi} / 6, e=0.1$
$\triangle f^{0}=\mathrm{pi} / 3, e=0.1$
* $f^{0}=\mathrm{pi} / 2, e=0.1$
$\begin{aligned} \bigcirc f^{0} & =\mathrm{pi} / 6, e=0.2 \\ +f^{0} & =\mathrm{pi} / 3, e=0.2 \\ \diamond f^{0} & =\mathrm{pi} / 2, e=0.2\end{aligned}$

(a)

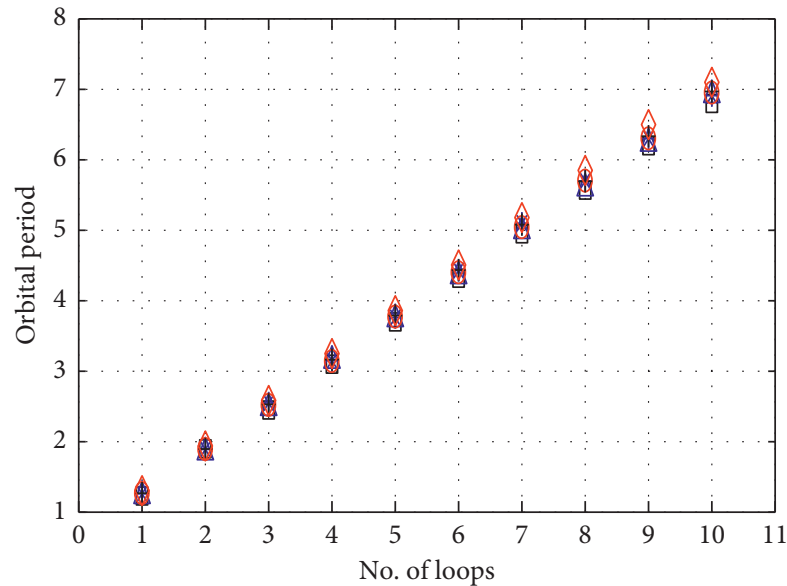
ㄱ $f^{0}=\mathrm{pi} / 6, e=0.1$
$0 f^{0}=\mathrm{pi} / 6, e=0.2$
$\Delta f^{0}=\mathrm{pi} / 3, e=0.1$
$+f^{0}=\mathrm{pi} / 3, e=0.2$
* $f^{0}=\mathrm{pi} / 2, e=0.1$
$\diamond f^{0}=\mathrm{pi} / 2, e=0.2$

(b)

Figure 12: Variation in the (a) initial position of periodic orbits and (b) orbital period when $\dot{y}^{0}=0.005$.

initial locations of periodic orbits for three different values of true anomaly come closer to each other as the number of loops increases from 1 to 10 . The variation in periods of periodic orbits with respect to the number of loops for three different values of true anomaly as $\pi / 6, \pi / 3$, and $\pi / 2$ is shown in Figure 12(b) with a velocity $(0,0.005,0)$. It has been seen in both cases of initial velocities and eccentricities that orbital period increases with increasing number of loops.

Figures 13(a)-13(c) show the variation in the initial position and period with respect to number of loops for two different eccentricities 0.1 and 0.2 with given initial true anomalies $\pi / 6, \pi / 3$, and $\pi / 2$, respectively. In these three figures, the velocity is considered as $(0,0.002,0)$. This gives information about the variation in the initial position and orbital period when the values of eccentricity are changed as shown in all figures. The sharp observation for the singleloop orbit depicts that, for the given value of true anomaly, as the eccentricity goes on increasing and the initial position of periodic orbit shifts towards $x^{0}=0$. It has also been observed that, moving in the direction from 1- to 10-loop orbit, differences in the initial position for a given value of true anomaly decreases with increasing eccentricity. The orbital period is conserved as the velocity increases for a given number of loops, eccentricity, and initial true anomaly.

It is observed that, with the ascending number of loops, the initial position of periodic orbits approaches to the origin 


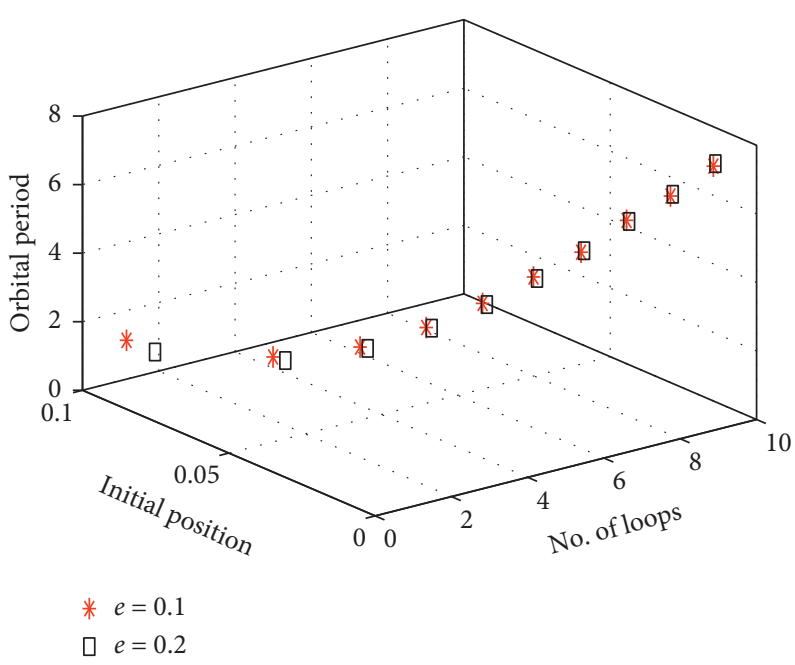

(a)

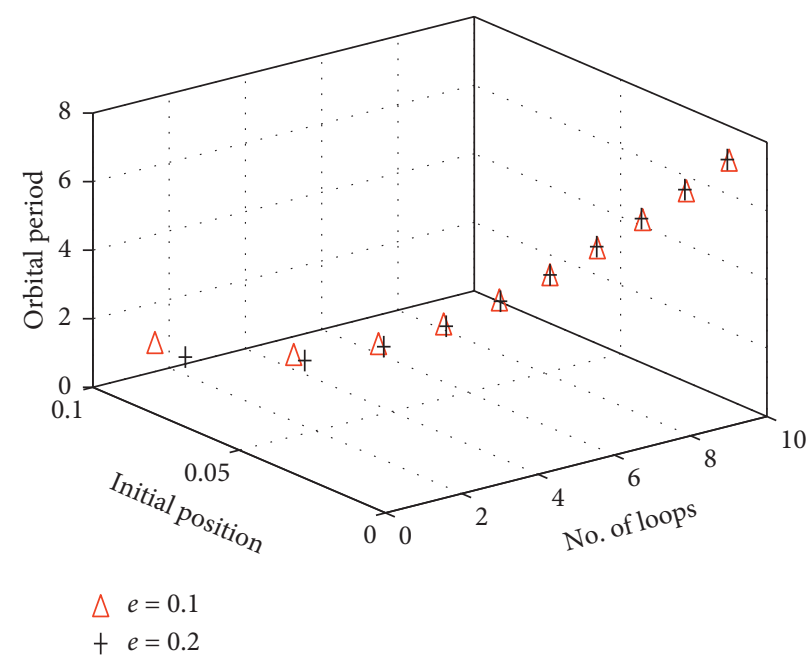

(b)

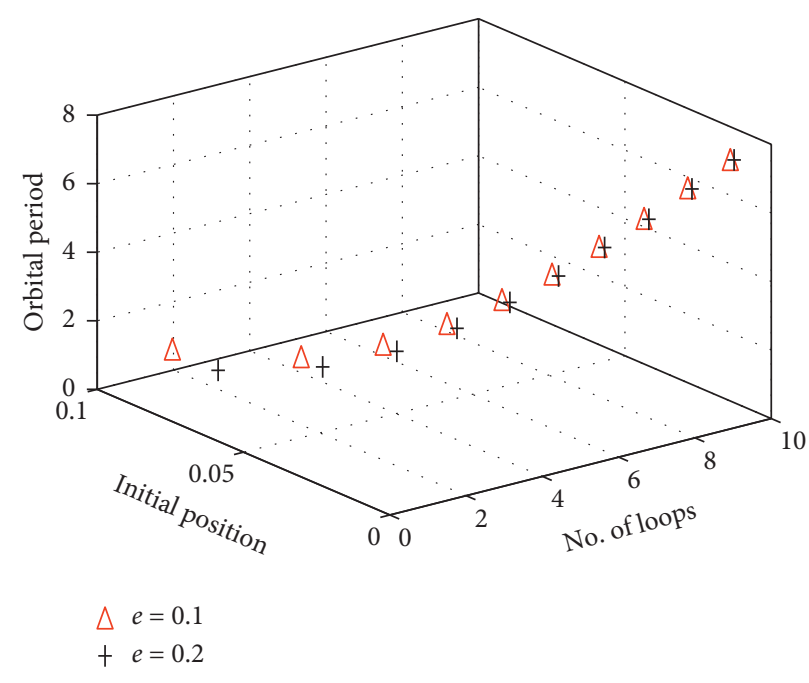

(c)

FIGURE 13: Variation in the initial position and orbital period, when velocity is $(0,0.002,0)$. (a) $f^{0}=\pi / 6$. (b) $f^{0}=\pi / 3$. (c) $f^{0}=\pi / 2$.

with an increasing value of orbital periods. When the eccentricity is 0.1 , for all the true anomalies, we get two-dimensional periodic orbits, but especially when true anomaly approaches to $\pi / 3$, the orbits becomes three-dimensional periodic and then again, as true anomaly approaches $\pi / 2$, the three-dimensional orbits becomes quasi in nature. Whereas when the eccentricity is 0.2 , we get two-dimensional periodic orbits for all the true anomalies. The nature of three dimensional orbits becomes highly quasi with an increasing value of the true anomaly. Thus, as the eccentricity increases, the nature of periodic orbits becomes more chaotic with an increasing value of true anomaly.

The periodic solution plays a vital role in the field of celestial mechanics. However, a solution feature in the form of mathematical expression for the behavior of the trajectory does not directly appear, but these features can be clearly shown when the solution is graphically represented. It also identifies the orbit whether it is loop or without loop. In case an orbit is having loops, the number of loops with internal position or external position can be determined easily with the same solution too. An orbital period of the orbit is the most important parameter that gives information about the time required to complete one revolution by the deputy satellite. The value of this parameter is determined easily by the periodic solution. It is evident from this study that, as the number of loops increases, the period of the periodic orbit increases. Periodic orbits with a lower number of orbital periods have been more emphasized in celestial mechanics. In this regard, the main focus of this study is 1- to 10-loop periodic orbit with a value of period less than 7 . In formation satellites, the initial true anomaly and initial velocity of deputy satellite play an important role which affects the initial position of periodic orbits of deputy satellite. Also, in the LVLH frame, the origin is located at the position of chief satellite, so the eccentricity of the orbit of chief satellite also affects the initial position and orbital period of deputy 
satellite. Thus, it is important to study the effect of these parameters on periodic orbit of deputy satellite.

\section{Conclusion}

The main focus of this work is on periodic orbit of deputy satellite in the elliptical case of formation satellites. The analysis of 1- to 10-loop periodic orbits with different values of initial true anomaly $f^{0}$ and eccentricity $e$ of the orbit of chief satellite has been performed by considering the initial position $\left(x^{0}, 0,0.0001\right)$ and initial velocity $\left(0, \dot{y}^{0}, 0\right)$. It has been observed that, as the number of loops ascends from 1 to 10 , there is an increment in the orbital period, and the initial position of periodic orbits approaches to origin. For a given number of loops, eccentricity, and initial true anomaly, the orbital period is conserved with regard as velocity ascends.

It is noticed that the effect of eccentricity and initial true anomaly on orbital period is negligible. For the given eccentricity $e$ and initial velocity $\left(0, \dot{y}^{0}, 0\right)$, as number of loops increases, the initial position $\left(x^{0}, 0,0.0001\right)$ shifts towards zero. It has also been concluded that, for the given number of loops, the effect of eccentricity and initial true anomaly on orbital period is negligible. Furthermore, with the given value of initial true anomaly $f^{0}$ and initial velocity $\left(0, \dot{y}^{0}, 0\right)$, the number of loops and increment in eccentricity $e$ and initial position $\left(x^{0}, 0,0.0001\right)$ approach the origin. Thus, to conclude with that for given value of true anomaly and eccentricity, there is a decrement in the value between the differences in initial position vectors with the ascending number of loops. In other words, for a given value of true anomaly and eccentricity, the difference in the initial position $(\Delta x, 0,0.0001)$ decreases as the number of loops increases.

Finally, we summarize and state that, in the frame work of formation satellite, 1-10 loop periodic orbits of deputy satellite are analyzed. In this analysis, there are three parameters, namely, initial true anomaly, initial velocity of deputy satellite, and eccentricity of orbit of chief satellite. The initial position and orbital period are investigated for these orbits.

It is observed that, with the ascending number of loops, the initial position of periodic orbits approaches to origin with an increasing value of orbital periods. When the eccentricity is 0.1 , for all the true anomalies, we get two-dimensional periodic orbits, but especially when true anomaly approaches to $\pi / 3$, the orbits become three-dimensional periodic and then, again as true anomaly approaches $\pi / 2$, the three-dimensional orbits becomes quasi in nature. Whereas when eccentricity is 0.2 , we get two-dimensional periodic orbits for all the true anomalies. The nature of three-dimensional orbits becomes highly quasi with an increasing value of true anomaly. Thus, as the eccentricity increases, the nature of periodic orbits becomes more chaotic with an increasing value of true anomaly.

It is remarkable that the orbital period remains unchanged for the given initial true anomaly, eccentricity, and number of loops with ascending velocity. Thus, the orbital period and nature of the periodic orbits are conserved with a negligible displacement in the initial position of the orbits with increasing velocity. It has also been observed that, for a given number of loops, eccentricity, and initial true anomaly with the increment in velocity, there is a decrement in the value of the initial position of the orbit. Thus, to conclude with that for a given value of true anomaly and eccentricity, there is a decrement in the value between the differences in initial position vectors with the ascending number of loops. Also, with the rise in velocity, there is negligible change in the value of differences in the initial position $(\Delta x, 0,0.0001)$ corresponding to each loop.

\section{Data Availability}

Data sharing is not applicable to this article as no datasets were generated or analyzed during the current study.

\section{Conflicts of Interest}

The authors declare that there are no conflicts of interest.

\section{References}

[1] F. Alzahrani, E. I. Abouelmagd, J. L. G. Guirao, and A. Hobiny, "On the libration collinear points in the restricted three-body problem," Open Physics, vol. 15, no. 1, pp. 58-67, 2017.

[2] E. I. Abouelmagd, J. L. G. Guirao, and J. Llibre, "Periodic orbits for the perturbed planar circular restricted 3-body problem," Discrete \& Continuous Dynamical Systems-B, vol. 24, no. 3, pp. 1007-1020, 2019.

[3] N. Pathak, V. Thomas, V. O. Thomas, and E. I. Abouelmagd, "The perturbed photogravitational restricted three-body problem: analysis of resonant periodic orbits," Discrete \& Continuous Dynamical Systems - S, vol. 12, no. 4-5, pp. 849-875, 2019.

[4] N. Pathak, E. I. Abouelmagd, and V. O. Thomas, "On higher order resonant periodic orbits in the photo-gravitational planar restricted three-body problem with oblateness," The Journal of the Astronautical Sciences, vol. 66, no. 4, pp. 475$505,2019$.

[5] J. L. G. Guirao, J. Llibre, and J. A. Vera, "Periodic orbits of Hamiltonian systems: applications to perturbed Kepler problems," Chaos, Solitons \& Fractals, vol. 57, pp. 105-111, 2013.

[6] R. M. Tudoran, "Asymptotic stabilization with phase of periodic orbits of three-dimensional Hamiltonian systems," Journal of Geometry and Physics, vol. 121, pp. 33-41, 2017.

[7] M. T. de Bustos, J. L. G. Guirao, J. Llibre, and J. A. Vera, "New families of periodic orbits for a galactic potential," Chaos, Solitons \& Fractals, vol. 82, pp. 97-102, 2016.

[8] J. Llibre and C. Vidal, "Periodic motion in non-axially symmetric galaxies," Journal of Geometry and Physics, vol. 140, pp. 1-9, 2019.

[9] O. Montenbruck, M. Wermuth, and R. Kahle, "GPS based relative navigation for the TanDEM-X mission-first flight results," Navigation, vol. 58, no. 4, pp. 293-304, 2011.

[10] R. S. Smith and F. Y. Hadaegh, "Distributed estimation, communication and control for deep space formations," IET Control Theory \& Applications, vol. 1, no. 2, pp. 445-451, 2007.

[11] C. W. T. Roscoe, S. R. Vadali, K. T. Alfriend, and U. P. Desai, "Satellite formation design in orbits of high eccentricity with performance constraints specified over a region of interest: 
mms phase II," Acta Astronautica, vol. 82, no. 1, pp. 16-24, 2013.

[12] B. Wu, G. Xu, and X. Cao, "Relative dynamics and control for satellite formation: accommodating $j_{2}$ perturbation," Journal of Aerospace Engineering, vol. 29, no. 4, Article ID 04016011, 2016.

[13] B. Wu, D. Wang, and E. K. Poh, "Decentralized sliding-mode control for attitude synchronization in spacecraft formation," International Journal of Robust and Nonlinear Control, vol. 23, no. 11, pp. 1183-1197, 2013.

[14] G. W. Hill, "Researches in the lunar theory," American Journal of Mathematics, vol. 1, no. 1, pp. 5-26, 1878.

[15] J. L. Junkins and H. Schaub, Analytical Mechanics of Space Systems, AIAA, Reston, VA, USA, 2003.

[16] S. A. Schweighart and R. J. Sedwick, "High-fidelity linearized J model for satellite formation flight," Journal of Guidance, Control, and Dynamics, vol. 25, no. 6, pp. 1073-1080, 2002.

[17] J. A. Roberts and P. C. E. Roberts, "The development of high fidelity linearized $J_{2}$ models for satellite formation flying control," in Proceedings of the 14th AAS/AIAA Space Flight Mechanics Meeting, Maui, HI, USA, February 2004.

[18] J. A. Roberts, Development of a Relative Motion Model for Satellite Formation Flying Around $L_{2}$, Cranfield University; School of Engineering, Cranfield, UK, 2004.

[19] Z. Wang and X. Chen, "Optimal maneuver technology for satellite formation flying," in Informatics in Control, Automation and Robotics, pp. 287-290, Springer, Berlin, Germany, 2011.

[20] M. A. Nurge, R. C. Youngquist, and S. O. Starr, "A satellite formation flying approach providing both positioning and tracking," Acta Astronautica, vol. 122, pp. 1-9, 2016.

[21] R. C. Youngquist, M. A. Nurge, and S. O. Starr, "Alternating magnetic field forces for satellite formation flying," Acta Astronautica, vol. 84, pp. 197-205, 2013.

[22] D. Ivanov, M. Ovchinnikov, and S. Shestakov, "Satellite formation flying control by mass exchange," Acta Astronautica, vol. 102, pp. 392-401, 2014.

[23] E. I. Abouelmagd, D. Mortari, and H. H. Selim, "Analytical study of periodic solutions on perturbed equatorial two-body problem," International Journal of Bifurcation and Chaos, vol. 25, no. 14, Article ID 1540040, 2015.

[24] E. I. Abouelmagd, S. Elshaboury, and H. Selim, "Numerical integration of a relativistic two-body problem via a multiple scales method," Astrophysics and Space Science, vol. 361, no. 1, Article ID 38, 2016.

[25] E. I. Abouelmagd, J. Llibre, and J. L. G. Guirao, "Periodic orbits of the planar anisotropic kepler problem," International Journal of Bifurcation and Chaos, vol. 27, no. 3, Article ID 1750039, 2017.

[26] E. I. Abouelmagd, "Periodic solution of the two-body problem by $\mathrm{KB}$ averaging method within frame of the modified Newtonian potential," The Journal of the Astronautical Sciences, vol. 65, no. 3, pp. 291-306, 2018. 